\title{
GAS CLOUDS FROM TIDALLY DISRUPTED STARS IN ACTIVE GALACTIC NUCLEI
}

\author{
Nico Roos \\ Sterrewacht Leiden, P.O. Box 9513, 2300 RA Leiden, The Netherlands \\ Received 1990 December 17; accepted 1991 July 18
}

\begin{abstract}
A direct implication of the presence of massive black holes in galactic nuclei is the occasional tidal disruption of a star in the vicinity of the hole. The tidal disruption rate will be enhanced as a result of perturbations of the star cluster around the hole (for instance during the final stage of a merger with a smaller galaxy) leading to prolonged periods of activity. Recent investigations of the tital break-up of solar-type stars near massive black holes have shown that debris gas will be distributed throughout the galactic nucleus. About half of the stellar debris mass is strongly bound to the hole and will quickly be swallowed while the rest is expelled with typical velocities of order $5000 M_{6}^{1 / 6} \mathrm{~km} \mathrm{~s}^{-1}$, where $M_{6}=M_{\text {hole }} / 10^{6} M_{\odot}$. In this paper the structure and properties of freely expanding (unbound) remnant clouds irradiated by the central continuum source of an AGN are explored. It is argued that the remnant cloud fragments into cold clumps of gas $\left(T \approx 10^{4} \mathrm{~K}\right)$ surrounded by hot gas at the Compton temperature $\left(T_{\mathrm{C}} \approx 10^{7-8} \mathrm{~K}\right)$ when the ionization parameter $\Xi$ (defined as the ratio of the ionizing radiation pressure to the gas pressure) reaches the critical value $\Xi_{c} \approx 10$. Subsequently the fraction of hot gas slowly increases at the expense of the cold component while the ionization parameter of both components remains equal to $\Xi_{c}$. Photoionization of cold gas in the outmoving and radially elongated remnant clouds produces a broad line region at a distance of $\approx 10^{16-17} \mathrm{~cm}$ in Seyferts and $\approx 10^{17-18} \mathrm{~cm}$ in quasars. The broad line region (BLR) has an outer boundary where the remnant clouds become optically thin to the ionizing radiation from the central source. The densities and column densities in the BLR are in the range $\sim 10^{9-11} \mathrm{~cm}^{-3}$ and $\sim 10^{24-27} \mathrm{~cm}^{-2}$, respectively $(\Xi \sim 10)$. Bound debris orbiting at $\sim 10^{15-16} M_{6}^{2 / 3} \mathrm{~cm}$ with characteristic densities $\geq 10^{11} \mathrm{~cm}^{-3}$ may contribute to the inner part of the broad line region yielding symmetric lines, whereas a relatively small number $(\sim 10-100)$ of large outmoving remnant clouds produces a more asymmetric and bumpy line profile. There are two potential problems with this new model for the BLR: First, a high tidal disruption rate of order $\geq 0.1 M_{\odot} \mathrm{yr}^{-1}$ seems required to replenish the gas in the BLR in Seyferts as well as in QSOs. Second, in some AGN the central continuum source may be obscured by optically thick remnant clouds along our line of site.

A very attractive property of the tidal disruption model is that it offers a natural interpretation not only for the broad emission lines but for a number of other features in AGN spectra as well. Tidal disruptions will provide cold, dense gas close to the central source which can reprocess the radiation from the central source and produce a thermal bump in the optical/UV as well as several characteristic X-ray features. Optically thin remnants moving along our line of site outside the BLR at about $10^{18-19} \mathrm{~cm}$ may produce broad absorption lines similar to those observed in broad absorption line QSOs. The radially outmoving remnants are decelerated and compressed by the interstellar medium at radii $\geq 10^{19} \mathrm{~cm}$, where they will emit narrow forbidden lines.
\end{abstract}

Subject headings: black hole physics - galaxies: kinematics and dynamics - galaxies: nuclei galaxies: Seyfert - quasars: general

\section{INTRODUCTION}

A typical spectrum of an active galactic nucleus (AGN) is often described as a nonthermal continuum on which several other components are superposed, such as broad and narrow emission lines, an infrared bump, a UV-bump and a soft X-ray excess (e.g., Sanders et al. 1989). The nonthermal continuum radiation presumably arises from within ten Schwarzschild radii of an accreting black hole having a mass of $\sim 10^{6}$ to $10^{9}$ $M_{\odot}$. The other components are thought to be produced by an accretion disk and/or through reprocessing of the primary radiation by relatively dense and cold $\left(T \sim 10^{4} \mathrm{~K}\right)$ gas (plus perhaps dust) around the central source.

The best testimony to the presence of cold, dense gas close to the central source is given by the broad emission lines. These lines are produced by gas with characteristic densities of $10^{8}$ $10^{11} \mathrm{~cm}^{-3}$ which appears to be moving with velocities $\sim 10^{4}$ $\mathrm{km} \mathrm{s} \mathrm{s}^{-1}$ at radii $10^{16}-10^{18} \mathrm{~cm}$ from the central source
(Osterbrock 1985). Guilbert \& Rees (1988) and Lightman \& White (1988) have argued that in accretion-driven AGN one would expect cold gas with even higher densities $\left(\geq 10^{14} \mathrm{~cm}^{-3}\right)$ to exist at radii smaller than $\sim 10^{15} \mathrm{~cm}$. They showed that this matter would modify and reprocess the nonthermal X-ray radiation from the central engine, which would be reemitted predominantly as black body radiation in the UV region (see also Ferland \& Rees 1988). This matter would also produce several features in the X-ray band such as characteristic bump between $1 \mathrm{keV}$ and $1 \mathrm{Mev}, \mathrm{X}$-ray absorption (flattening the spectrum below $\sim 10 \mathrm{keV}$ ), ionization edges and fluorescence lines (in particular Fe K-shell). Such features have recently been detected in the spectra of several Seyferts by the X-ray satellites Ginga and EXOSAT (Matsuoka et al. 1990; Nandra et al. 1989).

The origin of cool, dense gas in AGN, which might also provide fuel for the central engine, is still a subject of specula- 
tion. Occam's razor suggests that we first should look for a single source. In this paper we will show that tidal disruption of stars near a central massive black hole provides a source of cool gas clouds with the above range of densities and radii. In particular we will show that the radii, densities, column densities and velocities of the cold gas in the remnant clouds produced by tidal disruption of solar-type stars are in the range required to explain the broad lines in AGN.

Gas from tidally disrupted stars was first considered by Hills (1975) as a possible fuel source for Seyferts and QSOs. A star of mass $m_{*}$ and radius $r_{*}$ (density $\rho_{*}$ ) is tidally disrupted by a black hole of mass $M_{h}$ when it passes within a tidal distance

$$
r_{T} \simeq\left(M_{h} / m_{*}\right)^{1 / 3} r_{*} \simeq 7 \times 10^{12} M_{6}^{1 / 3}\left(\rho_{*} / \rho_{\odot}\right)^{1 / 3} \mathrm{~cm}
$$

of the hole, where $M_{6}$ is the black hole mass in $10^{6}$ solar masses and $\rho_{\odot}$ is the mean solar density. Note that this close to the Schwarzschild radius $r_{S}=3 \times 10^{11} M_{6} \mathrm{~cm}$. One of the attractive features of this process is that the bound debris gas has a very low angular momentum and it is not necessary to have a large viscous accretion disk to bring the matter close to the hole. There are, however, two problems connected with the luminosity of the most powerful QSOs. Firstly, $r_{T}$ becomes smaller than $r_{S}$ when the black hole mass exceeds a critical mass of a few times $10^{8} M_{\odot}$. Stars will be swallowed whole by such massive black holes. There are good reasons for assuming that the masses of black holes in galactic nuclei do not exceed $\sim 10^{9} M_{\odot}$ (Rees 1990). However, some QSOs at high redshifts appear to have bolometric luminosities of $\sim 10^{48} \mathrm{ergs}^{-1}$. Such QSOs cannot be fueled by tidal disruptions of main sequence stars if they are radiating isotropically at the Eddington limit, which would imply a mass exceeding the critical mass for tidal disruption. Hills (1978) already suggested that the decrease in tidal disruption efficiency when black holes become larger than a few times $10^{8} M_{\odot}$ may be related to the difference between the radio-quiet and radio-loud $A G N$ (see $\S 5$ ). The second problem is the high tidal disruption rate required to fuel AGN. The energy output of a massive black hole which is accreting mass at a rate $\dot{M}$ is

$$
L=6 \times 10^{45}\left(\frac{\eta}{0.1}\right)\left(\frac{\dot{M}}{1 M_{\odot} \mathrm{yr}^{-1}}\right) \operatorname{ergs~s}^{-1},
$$

where $\eta$ is the mass to energy conversion efficiency. The most powerful QSOs may require accretion rates of $\sim 100 M_{\odot}$ $\mathrm{yr}^{-1}$. The tidal disruption rate in normal (unperturbed) galactic nuclei is limited by the slow diffusion via two-body relaxation of stars into (low angular momentum) loss-cone orbits (Lightman \& Shapiro 1977; Frank \& Rees 1976). During the formation and rapid early evolution of galactic nuclei stellar tidal disruptions may have been important for the growth of massive black holes and for the power output of high-redshift QSOs. However, tidal disruptions will be unable to sustain the activity of powerful AGN at later epochs unless the stellar orbits in the galactic nucleus are somehow perturbed more efficiently than via stellar encounters (Shields \& Wheeler 1978). This led Roos (1981, hereafter paper I) to suggest that infall and accretion (merging) of a smaller galaxy would, during the final stage of the merger, perturb the stellar orbits around a central massive hole sufficiently to replenish the loss-cone and boost the tidal disruption rate by a few orders of magnitude to about $1 M_{\odot} \mathrm{yr}^{-1}$. Such a disruption rate may be sufficient for a typical QSO, but not for the most powerful QSOs having luminosities of $10^{4-48}$ ergs $\mathrm{s}^{-1}$. Note that this problem and the previous one would be less severe if the energy output of the most powerful QSOs had been overestimated, for instance due to Doppler beaming of their (optical) continuum.

A central question in the tidal disruption model, which has been discussed by several authors over the past years (e.g., Lacey, Townes, \& Hollenbach 1982; Rees 1982; Sanders 1984), is the following: what is the fate of the debris gas? In order to answer this question we first need to know the distribution of binding energies (to the hole) of the stellar debris just after the star has been broken up. This was recently investigated by Rees (1988) and Evans \& Kochanek (1989). Their main results, which we will briefly discuss below, form the basis for the investigation presented in this paper.

The mean specific binding energy of debris gas from stars on parabolic orbits will be equal to $-v_{*}^{2}=-G m_{*} / r_{*}$, the specific internal binding energy of the original star. Hills (1978) assumed that most of the debris gas would have this binding energy and argued that line radiation from the debris cloud would account for the narrow line region. Later investigations by Lacey et al. (1982) and by Rees (1988) have shown that the distribution of binding energies must be very broad. Rees (1988) argued that just before tidal break-up of the star at pericenter the strong nonlinear quadrupole distortion traveling over the star causes the star to rotate approximately synchronously with its orbital motion around the hole. The tidal bulge facing the hole would then have a velocity $v_{\text {peri }}$ $-\Delta v$, where $v_{\text {peri }}$ is the orbital velocity at pericenter, while the opposite side of the star has a velocity $v_{\text {peri }}+\Delta v$, where $\Delta v=$ $v_{*}=\left(M_{h} / m_{*}\right)^{-1 / 3} v_{\text {peri }}$. As a result the stellar remnant will have a wide range of specific binding energies (to the hole) extending from $\epsilon_{\min } \simeq-v_{*}^{2}-v_{0}^{2}$ to $\epsilon_{\max } \simeq-v_{*}^{2}+v_{0}^{2}$ where

$$
v_{0} \simeq\left(M_{h} / m_{*}\right)^{1 / 6} v_{*} \simeq 5000 M_{6}^{1 / 6}\left(m_{*} / M_{\odot}\right)^{1 / 3}\left(r_{*} / R_{\odot}\right) \mathrm{km} \mathrm{s}^{-1} .
$$

Gas from tidally disrupted stars will thus be distributed throughout the whole nucleus from $r_{T}$ to greater than $1 \mathrm{pc}$. Evans \& Kochanek (1989) have recently done a numerical simulation of the tidal break-up of a solar type star by a $10^{6}$ $M_{\odot}$ black hole. They confirm the above results and find $v_{0} \sim$ $7000 \mathrm{~km} \mathrm{~s}^{-1}$. Since $v_{0}^{2}$ is much larger than $v_{*}^{2}$, and also much larger than the orbital energy of the original star, about half of the stellar remnant mass will be strongly bound to the hole, with orbital velocity $v_{0}$, while the other half will be expelled from the nucleus. The stellar remnant expands adiabatically as it moves outward with expansion velocity $\sim v_{*}$. The bound fraction of the cold and still dense gas turns around at $r \geq r_{0}$, which is given by

$$
\begin{aligned}
r_{0}=G M_{h} / v_{0}^{2} & \simeq r_{*}\left(M_{h} / m_{*}\right)^{2 / 3} \simeq r_{T}\left(M_{h} / m_{*}\right)^{1 / 3} \\
& \simeq 7.10^{14} M_{6}^{2 / 3}\left(m_{*} / M_{\odot}\right)^{-2 / 3}\left(r_{*} / R_{\odot}\right) \mathrm{cm}
\end{aligned}
$$

At a time

$$
T_{0}=\frac{\pi}{\sqrt{2}} \frac{r_{0}}{v_{0}} \sim 0.1 M_{6}^{1 / 2} \mathrm{yr}
$$

after disruption the bound gas returns to $r_{T}$ where it starts forming an accretion torus. The rate at which the bound debris is accreted by the hole is determined by the circularization time scale and the time scale for the evolution of the viscous torus at $r_{T}$. If both time scales are short the accretion rate will be comparable to the rate at which the debris is falling back to $r_{T}$, giving rise to an energetic flare with characteristic decline time 
$T_{0}$ (Rees 1988). If one or both time scales exceed the orbital time scale the energy output will be spread out more evenly in time (Canizzo, Lee, \& Goodman 1990).

Tidal debris not only provides a source of fuel for the central engine, but it will also imprint characteristic features upon the spectra of AGN by reprocessing radiation from the central source. It was already pointed out by Shields (1989) that the characteristic velocity of tidal debris given in equation (1.3) is remarkably similar to the typical velocities of the broad line gas. The mean density and temperature of the gas are also similar. Immediately after disruption the stellar remnant expands adiabatically and the temperature rapidly drops as the thermal energy of the original star is converted into energy of expansion (expansion velocity $\sim v_{*}$ ). The pressure in the remnant cloud is sufficient to ensure that its expansion will not be hampered by the interstellar medium in the inner parsec of the galaxy. Assuming spherical expansion of remnant clouds we find that the mean density of the remnant cloud is given by

$$
\bar{n}(r) \sim n_{\odot}\left(\frac{v_{*} r}{v_{0} r_{*}}\right)^{-3} \sim 3 \times 10^{14} M_{6}^{1 / 2} r_{15}^{-3} \mathrm{~cm}^{-3}
$$

where $r_{15}=r / 10^{15} \mathrm{~cm}$.

These results invite further investigation of the properties and observable effects of gas from tidally disrupted stars in AGN. In this paper some results of an explorative investigation will be reported. First, in $\S 2$, we discuss fuelling of the central hole in galactic nuclei during periods of enhanced tidal disruptions induced by galaxy mergers. In $\S 3$ we investigate the physical conditions in stellar remnants that are irradiated by the central continuum source of an AGN. In $\S 4$ the properties of the broad line region produced by debris clouds are discussed and compared with the properties inferred from the observations. In $\S 5$ we briefly discuss some other characteristic features in AGN spectra in the context of the tidal disruption model, such as the big blue bump, X-ray characteristics, broad absorption lines in QSOs, the narrow line region, and the difference between radio-loud and radio-quiet AGN. The main results are summarized in $\S 6$.

\section{STARS AS FUEL FOR AGN}

\subsection{Galaxy Mergers and Recurrent Activity}

The question of the origin and growth of black holes in galactic nuclei is connected with the evolutionary history of galaxies and their nuclei. One approach to this problem is to assume that galaxies form and evolve in relative isolation. Within this concept it is natural to assume that only a small $(\sim 1 \%)$ subset of priviliged galaxies has been able to form massive black holes in their nuclei and sustain QSO activity at $z \sim 2$, evolving into Seyfert activity at the present epoch. The fuel source could be gas lost from stars through stellar collisions or stellar evolution. Tidal disruption of stars may be less important due to depletion of stars on loss-cone orbits (e.g., Murphy, Cohn, \& Durisen 1990). Another approach which has gained much popularity within the last 10 years, is to regard galaxy formation as part of a hierarchical clustering process as in the cold dark matter theory (Blumenthal et al. 1984). Galaxy formation is then not a single collapse event but rather a sequence of merger events with decreasing frequency. Galaxy mergers at the present epoch must be regarded as the tail of the galaxy formation process marking the transition from the epoch of dissipational galaxy formation to the epoch of dissipationless group and cluster formation. In such a scenario it is more natural to assume that the nuclei of all bright galaxies (at $z=0$ ) harbor massive black holes and that activity is a recurrent phenomenon which is triggered by galaxy mergers. The observed fraction of Seyferts among galaxies would then be equal to the (present) duty cycle $\left(\Delta t_{\text {on }} / \Delta t_{\text {off }} \sim 10^{-2}\right)$ for activity in the nuclei of galaxies, which is consistent with the observational estimates of the Eddington ratio for local AGN (Cavalieri \& Padovani 1988). Other reasons for preferring this approach (recently summarized by Rees 1990) are the following: (1) there is some evidence that the nuclei of nearby galaxies contain massive compact objects; (2) the total luminosity of QSOs per comoving volume integrated over the Hubble time would yield black hole masses that are too large if they were to reside in only $\sim 1 \%$ of all bright galaxies. The rapid cosmological evolution of powerful AGN suggests that the interaction rate among galaxies has also evolved considerably since a redshift of about 2. Several independent observational studies have recently provided evidence in favor of such a rapid evolution (Zepf \& Koo 1989; Lonsdale et al. 1990; Maddox et al. 1990). The evolution of the merger rate depends upon the galactic environment. It is steepest for galaxies in rich clusters, suggesting that the steep evolution of powerful AGN is due to the rapid formation and evolution of first-ranked galaxies in rich clusters, where the merging rate drops rapidly during the collapse and virialization of the cluster (Roos 1985b; Efstathiou \& Rees 1988; Carlberg 1990).

Several mechanisms have been proposed through which galaxy interactions and mergers could trigger activity in galactic nuclei. They may provide fresh fuel for the central engine by causing a flow of gas towards the galactic center (Noguchi 1988; Lin, Pringle, \& Rees 1988; Hernquist 1989) and/or a burst of star formation (Norman \& Scoville 1988). In hierarchical clustering scenarios the relative velocities of interacting galaxies outside rich clusters is low and dynamical friction is strong enough to ensure merging of the galaxies within a few orbital periods. If the nucleus of the secondary galaxy is sufficiently compact it will sink to the nucleus of the primary galaxy where it will cause activity by boosting the disruption rate of stars near the hole (paper I).

In previous papers (paper I; Roos 1985a, b) an attempt was made to estimate the local luminosity function of nuclei in which the tidal disruption rate was enhanced due to a merger. It was noted that small galaxies are probably most effective in triggering activity providing they contain nuclei that are dense enough to resist the tidal field in the central part of the parent galaxy. M32, the companion galaxy of M31 might be a good example. Its central density is comparable to that in the central part of M31 and it may even contain a compact central object with a mass $\sim 5 \times 10^{6} M_{\odot}$ (Tonry 1987). Such companion galaxies are more numerous than larger galaxies and they will perturb the nucleus of the parent galaxy during a longer time since the dynamical friction time is inversely proportional to their mass. Shields \& Wheeler (1978) estimated that large-scale fluctuations of only $\sim 1 \%$ in the stellar density around a massive black hole $\left(M \sim 10^{8} M_{\odot}\right.$, stellar density $\sim 10^{7} M_{\odot}$ $\mathrm{pc}^{-3}$ at $1 \mathrm{pc}$ ) can boost the tidal disruption rate to $v_{\text {tid }} \sim 1 M_{\odot}$ $\mathrm{yr}^{-1}$. A secondary nucleus spiraling at $\sim 10 \mathrm{pc}$ from the hole could sustain this level of tidal disruptions during several million years if its mass was $\sim 10 \%$ of the total mass within 10 pc.

Bahcall \& Tremaine (1988) estimated that typical bright galaxies have gained about $30 \%$ in mean luminosity since a redshift of $\sim 0.7$ by capturing satellite galaxies. This is consistent 
with the accretion of a few satellite galaxies (mass ratio 0.1 ) in $10^{10} \mathrm{yr}$, yielding a duty cycle of $\sim 10^{-3}$ for powerful AGN having $v_{\text {tid }}=1$ and larger duty cycles for lower tidal disruption rates.

The estimates made above are very crude and tentative due to the many uncertainties regarding the structure of galactic nuclei, the merging rate among galaxies and the enhancement of the tidal disruption rate due to perturbations (or nonaxisymmetric distortions) of the central star distribution. The fuelling rate and duty cycle of powerful $\mathrm{AGN}$ may well require a more abundant source of fuel than gas from tidally disrupted stars. However, the presence of massive black holes in galactic nuclei naturally leads to the occasional destruction and subsequent accretion of stars in unperturbed galaxies and to prolonged periods of enhanced disruption frequency in disturbed nuclei. It is therefore interesting to investigate the observable characteristics of tidal disruptions in normal as well as in active galactic nuclei.

\subsection{Fuelling the Central Engine}

The duty cycle defined above pertains to the activity averaged over a period much longer than the mean time between successive disruptions. During an active period the level of activity may be quite variable if the mean time between successive disruptions is longer than the time scale for consumption of bound debris. In normal, unperturbed galactic nuclei containing massive black holes, for example, we expect tidal disruptions to occur every $10^{3-4}$ yr. Rees (1978) has pointed out that such events will be followed by a short flare lasting perhaps only a few years if the matter falling back to the hole is rapidly consumed. The rate at which bound debris from a single remnant falls back to $r_{T}$ is determined by the disruption of (specific) binding energies (to the hole). For a solar-type star which is broken up at $r_{T}$ this is given by Evans \& Kochanek (1989, hereafter E\&K):

$$
\frac{d m}{d \epsilon}=\left\{\begin{array}{ll}
\frac{1}{2} m_{*} / v_{0}^{2} & |\epsilon|<v_{0}^{2} \\
0 & |\epsilon|>v_{0}^{2}
\end{array} .\right.
$$

The amount of debris mass having apocenter distance smaller than $r$ is given by

$$
m(<r)=\frac{1}{2} m_{*}\left(1-r_{0} / r\right),
$$

so most bound matter turns around at radii $\geq r_{0}$. At a time $t$ $\left(\geq T_{0}\right)$ after disruption bound debris arrives at $r_{T}$ at a rate

$$
F=\frac{d m}{d t}=\frac{1}{3} \frac{m_{*}}{T_{0}}\left(\frac{t}{T_{0}}\right)^{-5 / 3}
$$

causing a short, intense flare if it is immediately consumed by the hole $(\dot{M}=F)$. In that case the accretion rate after each disruption event quickly rises to a maximum $\dot{M}_{\max }=\frac{1}{3} m_{*} / T_{0}$, followed by a decline as $\left(t / T_{0}\right)^{-5 / 3}$. After a time $\sim v_{\text {tid }}^{-1}$, the accretion of matter from the last disruption event is comparable to the accretion of matter from previous events, which is about $\dot{M}_{\text {max }}\left(v_{\text {tid }} T_{0}\right)^{5 / 3}$. Thereafter the accretion rate declines as $t^{-2 / 3}$ until the next disruption takes place.

There are several reasons why the energy output from a tidal disruption event could be more evenly distributed in time: (1) The circularization time, which may be defined as the time required for the bound debris to settle in an accretion torus at $r_{T}$, may be longer than the orbital time $T$. E\&K estimate it to be at least $\sim 10 T$; (2) The evolution time of the viscous torus may be longer than the circularization time (Canizzo et al.
1990); (3) The energy output attains the Eddington limit $\left(L_{\text {Edd }}=1.3 \times 10^{44} M_{6}\right.$ ergs s$\left.^{-1}\right)$ at an accretion rate

$$
\dot{M}_{\text {Edd }}=0.02 \eta_{0.1}^{-1} M_{6} M_{\odot} \mathrm{yr}^{-1},
$$

where $\eta_{0.1}=\eta / 0.1$ and $M_{6}=M / 10^{6} M_{\odot} \cdot \dot{M}_{\max }$ exceeds $\dot{M}_{\text {Edd }}$ for black hole masses smaller than $3.10^{7} M_{\odot}$. The energy output may then regulate the accretion rate so that $\dot{M} \sim \dot{M}_{\text {Edd }}$, while the excess bound debris is stored for later consumption. The maximum tidal disruption rate near massive black holes in galactic nuclei is determined by the maximum density of stars around the hole, which is set by stellar collisions (e.g., Frank \& Rees 1976). Note that this maximum rate may depend only weakly on black hole mass (paper I), so that the amount of stored fuel in well-fed AGN may increase with decreasing black hole mass.

There are several time scales in the tidal disruption model which are short enough to be interesting for variability studies. The shortest time scales are determined by the properties of the small accretion torus at $r_{T}$ (e.g., dynamical time $r_{T} / v_{T} \sim r_{*} / v_{*}$, sound travel time and light travel time) and by the physics of the accretion process. Over larger periods of time the variations in the light curve will be determined by the stochastic disruption of stars and the time scale for consumption of the bound debris via circularization and viscous evolution of the accretion torus. The variations in energy output will be large if the latter time scales are shorter than $v_{\text {tid }}^{-1}$.

\section{STRUCTURE AND PROPERTIES OF STELLAR REMNANTS}

In this section we discuss the properties of gas from solartype stars that are disrupted at the tidal disruption radius near a massive $\left(10^{6}-10^{8} M_{\odot}\right)$ black hole in an AGN. Solar-type stars are presumably the main source of debris gas for black holes in that mass range. White dwarfs are swallowed whole by black holes more massive than $\sim 10^{6} M_{\odot}$. Such stars will contribute to the mass of the hole but not to the amount of debris gas around the hole. Red giant atmospheres are stripped at a radius of about 100 times the tidal disruption radius of mainsequence stars. However, the fraction of such stars in a galactic nucleus with high stellar density will be very low due to stellar collisions. Red giants will therefore not be an important source of tidal debris mass. Most tidal disruptions of solar-type stars are expected to occur at pericenter distance between $\sim 1.1 r_{t}$ and $\sim 0.5 r_{T}$ (Carter \& Luminet 1985; E\&K). Partial disruptions and tidal capture of stars may occur at somewhat larger pericenter distances. General relativistic effects and possibly explosive release of nuclear energy may effect the outcome of encounters with smaller pericenter distance (Luminet \& Carter 1986). We will ignore such effects and study the fate of a debris cloud from a solar-type star that is disrupted at $r_{T}$. To simplify the discussion we adopt a value of $m_{*}=1 M_{\odot}, v_{*}=500 \mathrm{~km} \mathrm{~s}^{-1}$ and $r_{*}=7 \times 10^{10} \mathrm{~cm}$ for the disrupted star. The results are readily scaled to different values and our conclusions do not depend on this choice.

\subsection{Adiabatic Expansion of Stellar Remnants}

After disruption the stellar remnant expands adiabatically with expansion velocity $\sim v_{*}$ as it flies away from the hole with initial velocity $\sim 10^{2} v_{*}$. We assume for the moment that the remnant expands freely ignoring processes which might effect its initial evolution such as collisions with debris from other stars, or heating by the central source. Initially, the differences in orbital energy in different parts of the cloud are not important and the expansion is approximately spherically sym- 
metric. The sudden isentropic expansion of a spherical gas cloud into vacuum is described in several textbooks on gas dynamics (e.g., Zel'dovich \& Raiser 1967). During a short initial phase of accelerated outflow due to thermal pressure, the Initial internal energy is converted to kinetic energy of radial motion. Thereafter, the flow approaches a self-similar pressurefree form in which the expansion velocity increases linearly with radius to a maximum value of $\sim 3 v_{*}$, and the density decreases with radius. The form of the density profile is established during the initial stage of the expansion. In the following discussion we will generally assume that the mean (lateral) expansion velocity is $v_{\exp } \sim v_{*}$. The mean radius and density of the remnant cloud at time $t$ after disruption may be estimated by

$$
R_{R}=v_{\text {exp }} t
$$

and

$$
n_{R}^{\mathrm{sph}}=n_{*}\left(\frac{R_{R}}{r_{*}}\right)^{-3} .
$$

The temperature decreases rapidly as

$$
T \sim 10^{7}\left(\frac{R_{R}}{r_{*}}\right)^{-3(\gamma-1)} \mathrm{K},
$$

where $\gamma$ is the ratio of specific heats. When the remnant reaches $r_{0}$ the remnant stretches out in the radial direction due to differences in orbital energy. Bound matter starts falling back to the hole while the rest keeps moving out. At radii $\gg r_{0}$ the velocity distribution of unbound matter is given by $d m=$ $m_{*} v d v / v_{0}^{2}$. Most unbound matter resides in the head of the remnant rushing away with velocity $v_{0}$, while the rest trails behind in a tail. The differences in radial velocity causes the remnant cloud to expand uniformly in the radial direction with $v\left(r_{t}\right) \propto r_{t}$, where $r_{t}$ is the radial distance to the hole measured along the tail. Note that this implies that the density also increases along the tail since $\rho\left(r_{t}\right) \propto d m / d r \propto v\left(r_{t}\right) \propto r_{t}$. The velocity in the tail increases from $v=0$ at $r_{t}=r_{\text {apo }}\left(\propto \epsilon^{-1}\right)$, the distance where matter is turning around, to $v_{0}$ at $r_{t} r_{R}$, the position of the head of the remnant. Using $r_{R}=v_{0} t$, or, expressing distances in $10^{17} \mathrm{~cm}$ and time after disruption in years,

$$
r_{R, 17}=0.15 M_{6}^{1 / 6} t_{\mathrm{yr}} .
$$

Using this equation and Kepler's third law we may express $r_{\text {apo }}$ as

$$
r_{\text {apo,17 }}=0.17 M_{6}^{2 / 9} r_{R, 17}^{2 / 3} .
$$

The velocity along the tail increases with $r_{t}$ as

$$
v_{t}=\left(\frac{r_{t}-r_{\text {apo }}}{r_{R}-r_{\text {apo }}}\right) v_{0},
$$

or $\sim\left(r_{t} / r_{R}\right) v_{0}$ for $r_{\text {apo }} \ll r_{R}$ (and $\left.r_{t}>r_{\text {apo }}\right)$. The lateral extent of the outward moving remnant is independent of radial position along the tail. It can be expressed as $R=r_{R} v_{\exp } v_{0}^{-1}$, or

$$
R_{17}=\frac{R}{10^{17} \mathrm{~cm}}=0.1 M_{6}^{-1 / 6}\left(\frac{v_{\text {exp }}}{v_{*}}\right) r_{R, 17} .
$$

The mean density (in $10^{10}$ atoms $\mathrm{cm}^{-3}$ ) at $r_{t}$ is

$$
\bar{n}_{10}\left(r_{t}\right) \sim 0.014 M_{6}^{1 / 2}\left(\frac{v_{\text {exp }}}{v_{*}}\right)^{-3}\left(\frac{r_{t}}{r_{R}}\right) r_{R, 17}^{-3} .
$$

The total column density of the remnant measured along the tail is $-\frac{1}{2} m_{*} / \pi R_{R}^{2}$, or, in $10^{24}$ atoms $\mathrm{cm}^{-2}$,

$$
N_{R, 24}=2 M_{6}^{1 / 3}\left(\frac{v_{\text {exp }}}{v_{*}}\right)^{-2} r_{R, 17}^{-2} .
$$

The covering fraction of the head of a remnant is equal to

$$
\Omega\left(r_{R}\right)=\frac{R^{2}}{4 r_{R}^{2}}=10^{-2} M_{6}^{-1 / 3}\left(\frac{v_{\text {exp }}}{v_{*}}\right)^{2} .
$$

Note that the covering fraction of the tail at $r_{t}$ is larger by a factor $\left(r_{t} / r_{R}\right)^{-2}$, whereas the column density measured at $r_{t}$ is smaller by the same factor.

\subsection{Heating by the Central Continuum Source}

The thermal equilibrium of gas irradiated by the intense central continuum source in AGN is determined by the ionization parameter which may be defined as the ratio of the ionizing photon pressure to gas pressure (Krolik, McKee, \& Tarter 1981, hereafter KMT)

$$
\Xi=\frac{1}{4 \pi r^{2} n c k T} \int_{v_{\mathrm{L}}}^{\infty} L_{v} d v
$$

where $r$ is the distance to the continuum source, $n$ is the gas density, $T$ the gas temperature, $k$ the Boltzmann constant, $c$ is the speed of light, $L_{v}$ is the differential luminosity, and $v_{\mathrm{L}}$ is the frequency at the Lyman limit. In the literature one often uses the ionization parameter defined as the ratio of the ionizing photon density to the gas density

$$
U=\frac{1}{4 \pi r^{2} n c} \int_{v_{\mathrm{L}}}^{\infty} \frac{L_{v}}{h v} d v .
$$

The relation between the two ionization parameters depends on the index of ionizing spectrum and on its upper limit (see Collin-Souffrin \& Lasota 1988). A "soft" spectrum dominated by a "UV/soft X-ray bump" with an index close to zero up to a cutoff energy $h v_{\text {cut }}$ of a few Ry leads to

$$
U \sim \frac{2 k T}{h v_{\text {cut }}} \Xi \sim 0.1 T_{4} \Xi
$$

whereas a "hard" spectrum without a UV bump and an index $\sim 1$ yields $U \sim 0.01 T_{4} \Xi$.

The equilibrium temperature as a function of ionization parameter has been calculated by KMT and by Krolik \& Kallman (1988). The main characteristics of the equation state are sketched in Figure 1. For $\Xi<\Xi_{c} \sim 10$ heating by a continuum source is balanced by cooling via collisional line excitation and the gas maintains an equilibrium temperature of $T \sim 10^{4} \mathrm{~K}$. For $\Xi>\Xi_{c}$ the gas is ionized and the temperature approaches the Compton temperature $k T_{C} \sim\langle h v\rangle$. The detailed form of $\Xi(T)$ for $\Xi \geq \Xi_{c}$ depends on the spectral energy distribution of the incident radiation. A "soft" spectrum dominated by a UV/soft X-ray bump yields an equation of state similar to curve $b$ or $c$.

The equations of state given in Figure 1 are applicable to expanding stellar remnants in AGN too since the cooling time is much smaller than the dynamical time. The ionization parameter of a free expanding stellar remnant increases linearly with $r_{R}$. Using equation (3.8) we find

$$
\Xi=4.1 \xi_{0.3} L_{43} M_{6}^{-1 / 2}\left(\frac{v_{\text {exp }}}{v_{*}}\right)^{3}\left(\frac{r_{t}}{r_{R}}\right)^{-3} r_{R, 17} T_{4}^{-1},
$$




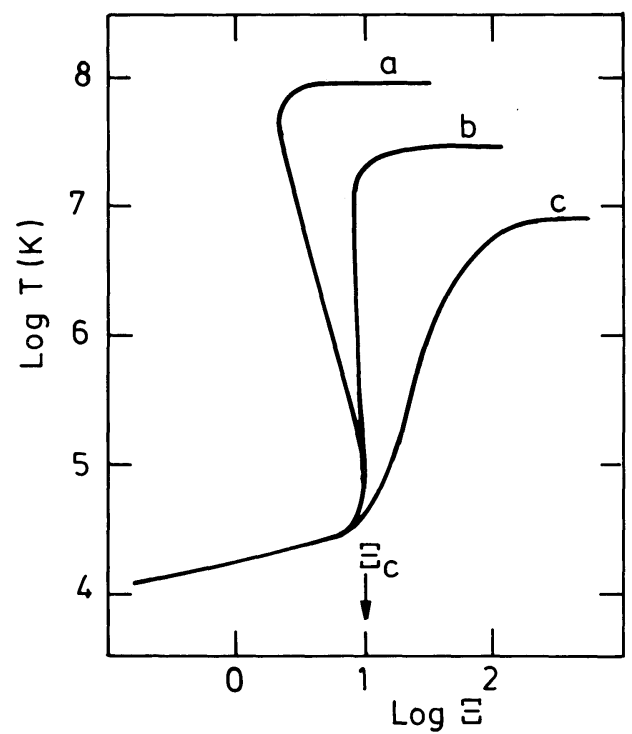

FIG. 1.-Schematic equation of state for gas irradiated by the central continuum source of an AGN (after Krolik et al. 1981). Curves $a, b$, and $c$ are for increasingly soft continuum spectra. Curves $a$ and $b$ are for pure power-law spectra with spectral index 1 and 1.5, respectively. Curve $c$ is for a power law $(\alpha \sim 1.5)$ plus a UV bump.

where $L_{43}$ is the bolometric luminosity of the continuum source in a $10^{43}$ ergs $\mathrm{s}^{-1}$. The quantity $\xi$ is the ratio of the ionizing luminosity over the bolometric luminosity; $\xi_{0.3}=\xi / 0.3$. The ionization parameter reaches the critical value $\Xi_{c} \sim 10$ at

$$
\left(\frac{r_{t}}{r_{R}}\right)^{-3} r_{R, 17}=2.4\left(\frac{v_{\text {exp }}}{v_{*}}\right)^{-3} \xi_{0.3}^{-1} L_{43}^{-1} M_{6}^{1 / 2} T_{4} \Xi_{c, 10} ;
$$

thereafter the mean temperature of the remnant cloud will start rising. A crucial point here is that the transition to the hot phase must take place under the constraint that $\Xi \geq \Xi_{c}$, i.e., the gas pressure should not exceed the ionizing radiation pressure divided by $\Xi_{c}$. Under this condition the temperature of a perfectly homogeneous cloud would rise in proportion with the cloud radius until it reaches the Compton temperature $T_{C}$. Debris clouds, however, are not homogeneous. On the large scale the density decreases in the lateral direction and in the direction of the tail. Small-scale inhomogeneities like sound waves will also be present. Such inhomogeneities cause the expanding remnant to fragment into two components: a hot component at the Compton temperature and a cold component with $T \sim 10^{4} \mathrm{~K}$. The temperature of slightly underdense regions will increase. This rise in temperature must be compensated by an increased expansion of that region in order to keep the ionization parameter $\geq \Xi_{c}$. The increased expansion is a local phenomenon. It is dynamically important on a scale which is set by the requirement that the expansion velocity on that scale is lower than the local sound speed in the gas. The expansion velocity on a scale $\Delta r$ is $\sim 500 \Delta r / R \mathrm{~km} \mathrm{~s}^{-1}$, where $R$ is the size of the remnant cloud, the sound speed in neutral gas is $\sim 10 T_{4}^{1 / 2} \mathrm{~km} \mathrm{~s}^{-1}$. We thus find

$$
\frac{\Delta r}{R} \leq 0.02 T_{4}^{1 / 2}
$$

The scale increases when the temperature of the gas rises. The increased expansion of the hot component reduces the expan- sion of the neighboring cold regions. It does not affect the expansion of the remnant cloud as a whole as long as the sound speed in the gas is lower than the expansion velocity of the remnant, $\sim 500 \mathrm{~km} \mathrm{~s}^{-1}$. The densities of the hot and cold components are both determined by the condition $\Xi=\Xi_{c}$ :

$$
\begin{aligned}
n_{\text {cold }}(r) & =\frac{\xi L_{\text {bol }}}{4 \pi c \Xi_{c} r^{2} k T_{\text {cold }}} \\
& =5.7 \times 10^{9} \Xi_{c, 10}^{-1} T_{4}^{-1} \xi_{0.3} L_{45} r_{17}^{-2} \mathrm{~cm}^{-3}
\end{aligned}
$$

and

$$
n_{\text {hot }}(r)=\frac{T_{\text {cold }}}{T_{\mathrm{C}}} n_{\text {cold }}(r),
$$

where $T_{\text {cold }} \sim 10^{4} \mathrm{~K}$ (see Fig. 2). The transition of cold gas into the hot phase lasts as long as the mean density in the remnant is

$$
n_{\text {hot }}(r)<\bar{n}(r)<n_{\text {cold }}(r) .
$$

As the ratio $\bar{n}(r) / n_{\text {cold }}$ declines the fraction of gas in the hot component increases at the expense of the cold component. This ratio can be written as

$$
n_{\text {cold }}(r) / \bar{n}(r)=\frac{f}{\epsilon}
$$

where $f$, the mass fraction of gas in the cold phase, and $\epsilon$, the

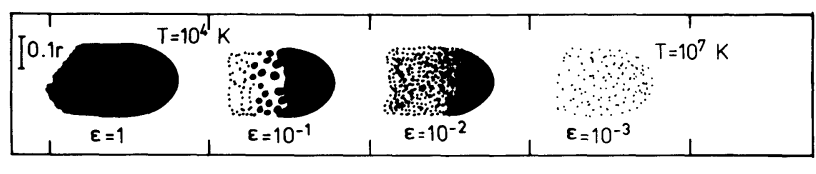

$$
\Xi\left\langle\Xi_{c} \vdash \quad \Xi=\Xi_{c} \times 10 \quad \longrightarrow \equiv \Xi_{c}\right.
$$

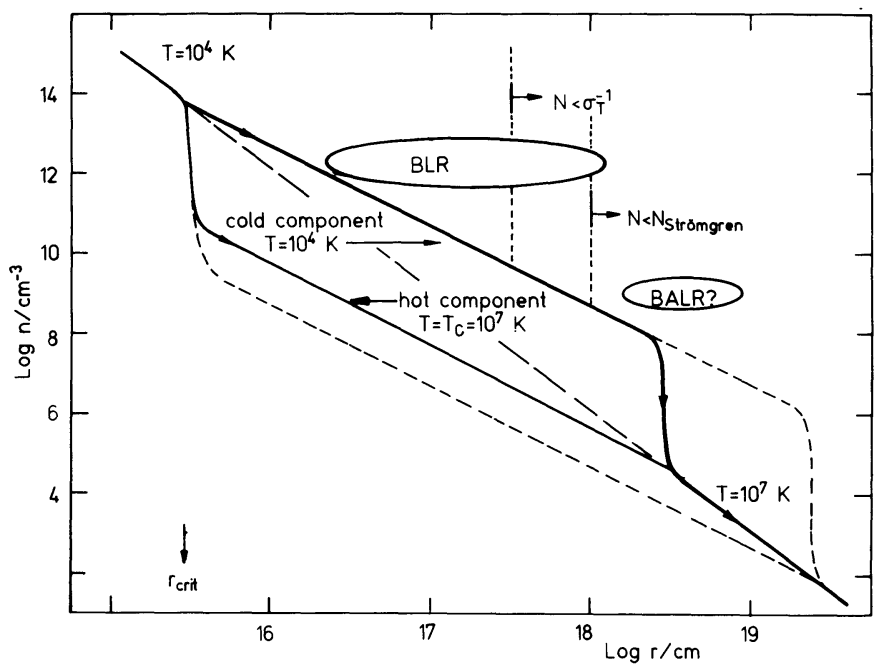

FIG. 2.-Gas densities in a freely expanding stellar remnant irradiated by the central continuum source of an AGN with bolometric luminosity $L_{\mathrm{bol}}=$ $10^{46} \mathrm{erg} \mathrm{s}^{-1}$ and $M_{\text {hole }}=10^{8} M_{\odot}$. At small radii the ionization parameter increases with radius until $\Xi=\Xi_{c}$ (see Fig. 1). Thereafter small-scale inhomogeneities are amplified and a small fraction of the remnant is heated to the Compton temperature (thin line: $T_{\mathrm{C}}=10^{7} \mathrm{~K}$; dashed line: $T_{\mathrm{C}}=10^{8} \mathrm{~K}$ ) while the rest stays at $\sim 10^{4} \mathrm{~K}$. The mean density is indicated by the long-dashed line. The densities of the cold and hot components are determined by the condition $\Xi \geq \Xi_{c} \sim 10$. Note that the remnant becomes optically thin to the ionizing radiation beyond $\approx 10^{18} \mathrm{~cm}$. The whole remnant is heated to $T_{\mathrm{C}}$ when $\bar{n}=n_{\text {hot }}$. 
fraction of the remnant volume occupied by cold matter must obey the relations

$$
f=1-\frac{T_{\text {cold }}}{T_{\mathrm{C}}} \frac{n_{\text {cold }}}{\bar{n}}
$$

and

$$
\epsilon=\frac{\bar{n}}{n_{\text {cold }}}-\frac{T_{\text {cold }}}{T_{\mathrm{C}}} .
$$

For unbound remnants moving out with constant velocity, $\bar{n} \propto R^{-3} \propto r^{-3}$ and $n_{\text {cold }} \propto n_{\text {hot }} \propto r^{-2}$, most of the matter remains in the cold component until the remnant has expanded by a factor $T_{\mathrm{C}} / T_{\text {cold }} \sim 10^{3-4}$ in radius and decreased by a factor $\sim 10^{9-12}$ in mean density! Condition (3.16) suggests that at the onset of the transition phase the remnant breaks up into a large number of cold fragments. Assuming spherical symmetry for remnant (radius $R$ ) and cold clouds (radius $r_{\mathrm{cl}}$ ) we find for the number of fragments or clouds

$$
\tilde{N}=\epsilon\left(\frac{R}{r_{\mathrm{cl}}}\right)^{3} \geq\left(0.02 T_{4}^{1 / 2}\right)^{3} \sim 10^{5}
$$

Note that the number of clouds is conserved during the subsequent expansion of unbound remnants moving with constant velocity since $r_{\mathrm{cl}} \propto n_{\text {cold }}^{-1 / 3} \propto r^{2 / 3}, R \propto r$ and $\epsilon \propto r^{-1}$.

Thus far, we have assumed that the remnant is heated uniformly. However, the column density of the remnants is still larger than $\sigma_{\mathrm{T}}^{-1}\left(\sigma_{\mathrm{T}}\right.$ is the Thomson cross section for electron scattering) at radii $r_{R}$ smaller than $\sim 1.4 \times 10^{17} M_{6}^{1 / 6} \mathrm{~cm}$. The incident radiation ionizes and heats the side of the remnant facing the continuum source. The extent of the ionized part of the remnant is determined by the Strömgren column density defined by

$$
N_{\mathrm{Str}}=\frac{U c}{\alpha_{\mathrm{B}}}
$$

The column density is measured from $r_{\text {apo }}$ radially outward along the remnant. The quantity $\alpha_{B}$ is the case B recombination coefficient, which is $2.6 \times 10^{-13} T_{4}^{0.8} \mathrm{~cm}^{3} \mathrm{~s}^{-1}$, and $U$ is the ionization parameter defined in (3.12), for cold matter in the remnant.

$$
U \sim(0.01-0.1) T_{4} \Xi=\zeta T_{4} \Xi .
$$

For unbound remnants $N_{\text {Str }}$ increases linearly with $r$ until $\Xi=\Xi_{c}$. Thereafter

$$
N_{\text {Str }}=2 \times 10^{23} \zeta_{0.05} \Xi_{c, 10} T_{4}^{0.2} \mathrm{~cm}^{-2} .
$$

The region behind the ionized region is shielded from the UV radiation. It is heated by X-rays (and higher energy photons) but it will have a lower equilibrium temperature. The whole remnant is ionized when $N_{R}<N_{\text {Str }}$, at a distance

$$
r_{\mathrm{Str}} \sim 5 \times 10^{17} \zeta_{0.05}^{-1 / 2} \Xi_{c, 10}^{-1 / 2} T_{4}^{-0.1}\left(\frac{v_{\text {exp }}}{v_{*}}\right)^{-1} M_{6}^{1 / 6} \mathrm{~cm}
$$

from the continuum source (Fig. 2).

The intense radiation from the central continuum source will exert dynamical effects on the debris clouds. The ionized part of a cool cloud is accelerated by the incident UV radiation. The acceleration is (e.g., Matthews \& Capriotti 1985, hereafter M\&C)

$$
g_{\mathrm{UV}}=\phi \frac{n \alpha_{\mathrm{B}}}{m_{\mathrm{H}}} \frac{h v_{\mathrm{L}}}{c} \sim 2\left(\frac{\phi}{2}\right) n_{10} T_{4}^{0.8} \mathrm{~cm} \mathrm{~s}^{-2},
$$

where $m_{\mathrm{H}}$ is the proton mass, $n$ is the gas density and $\phi$ is a constant depending on the chemical composition of the gas ( $\phi \sim 2$ for solar abundance). The radiative force pushes the ionized part of an optically thick cloud into the neutral part of the cloud where it is decelerated by ram pressure. We may obtain an estimate of the velocity $\Delta v$ of the ionized layer with respect to the neutral gas by putting $g_{\mathrm{UV}}$ equal to the acceleration due to ram pressure

$$
g_{\mathrm{ram}}=\frac{\bar{n}(\Delta v)^{2}}{N_{\mathrm{Str}}}
$$

yielding $\Delta v \approx 60 \mathrm{~km} \mathrm{~s}^{-1}$. Cold, optically thin cloudlets in the ionized part of the remnant are accelerated whole with respect to the hot component in the remnant. Their terminal velocity with respect to the hot gas is larger than $\Delta v$ estimated above by a factor $\left(n_{\text {cold }} / \bar{n}\right)^{1 / 2}\left(N_{\mathrm{cl}} / N_{\mathrm{Str}}\right)^{1 / 2}<\left(T_{\mathrm{C}} / T_{\text {cold }}\right)^{1 / 2} \approx 30$, yielding $\Delta v<2000 \mathrm{~km} \mathrm{~s}^{-1}$. The dynamical evolution of the decelerated gas is complicated. The gas is compressed in a shock front when its velocity relative to the neutral gas exceeds the internal sound speed. The compression will in turn increase the radiative acceleration of the gas which becomes Rayleigh-Taylor unstable at the decelerated side. The outcome of all these effects is uncertain, but it seems likely that they will amplify inhomogeneities in the gas and help to establish a two-phase medium when the ionization parameter reaches the critical value.

Acceleration by UV radiation is most important for highdensity gas close to the central source. At larger distances acceleration by soft X-rays (proportional to the metal abundance) may play a role. The acceleration of a cloud with hydrogen column density $N_{\mathrm{H}}$ and solar abundance at a distance $r$ of a source of soft X-rays with luminosity $L_{s \mathbf{x}}$ is

$$
g_{\mathrm{sx}} \approx 10^{36} \frac{L_{\mathrm{sx}, 45}}{r^{2}} \frac{N_{0}}{N_{\mathrm{H}}},
$$

for $N_{\mathrm{H}}>N_{0}=2.10^{20} \mathrm{~cm}^{-2}(\mathrm{M} \& \mathrm{C})$. The resulting velocity will be smaller than $\left(g_{\mathrm{sx}} r\right)^{1 / 2} \sim 120\left(L_{\mathrm{sx}, 45} r_{17}\right)^{1 / 2} M_{8}^{-1 / 6} \mathrm{~km} \mathrm{~s}^{-1}$.

The main conclusion from this section is that gas clouds produced by tidal disruption of stars contain cold and dense gas over a wide range in radii and densities throughout the nucleus of an active galaxy. The pressure of the cold gas in the expanding remnant clouds is fixed by the condition that the ionization parameter is equal to $\Xi_{c} \sim 10$.

\subsection{Structure of Unbound Debris Clouds}

Cold gas in outmoving remnants emits broad emission lines when it is photoionized by the central source. In this section we will study the structure of individual remnants in more detail. The results will be used in $\S 4.1$ to estimate the main properties of the BLR produced by outmoving remnants.

In $\S 3.1$ it was noted that at $r>r_{0}$ the outmoving debris cloud becomes elongated in the radial direction as a result of difference in orbital energy within the cloud. Strictly speaking not all the mass in the outmoving cloud is unbound. The specific orbital energy decreases toward the tail of the remnant and the tip of the tail contains some bound matter. At any moment the density and radial velocity increase linearly in the outward radial direction along the tail (see eqs. [3.8] and [3.6]). The remnant as a whole is freely expanding and so the density is decreasing everywhere as a function of time. In the discussion below we will assume that the lateral expansion velocity $v_{\text {exp }}$ equals $v_{*}$. The tail of the remnant begins to break 
up into cold clouds embedded in hot gas when the ionization parameter reaches the critical value $\sim 10$. This happens when the mean density $\bar{n}\left(r_{t}\right)=n_{\text {cold }}\left(r_{t}\right)$ at the radius given in equation (3.15). Thereafter most of the mass is in the cold phase with density $n_{\text {cold }}$ given by equation (3.17), until the mean density (eq. [3.8]) becomes lower than $n_{\text {cold }} T_{\text {cold }} / T_{\mathrm{C}}$. From this condition we find that the tail of the remnant is heated to the Compton temperature at radii smaller than

$$
r_{\text {hot }, 17}=0.16 r_{R, 17}^{4 / 3} L_{44}^{1 / 3} M_{6}^{-1 / 6}\left(\frac{\xi_{0.3}}{\Xi_{c, 10} T_{\mathrm{C}, 7}}\right)^{1 / 3},
$$

where $r_{R, 17}$ is the position of the head of the remnant given in equation (3.4). The ionized region of the tail extends to the Strömgren radius $r_{\text {Str }}$ defined as the distance along the tail where the column density (measured in the outward radial direction) equals the Strömgren column density given in equation (3.26). The column density at $r_{t}$ is proportional to $\left(r_{t}-r_{\text {apo }}\right)^{2} /\left(r_{R}-r_{\text {apo }}\right)^{2}$, and can be approximated by

$$
N\left(r_{t}\right) \sim\left(r_{t} / r_{R}\right)^{2} N\left(r_{R}\right)
$$

for $r_{t} \gg r_{\text {apo }}$. The Strömgren radius can be approximated by

$$
r_{\mathrm{Str}} \sim r_{\mathrm{apo}}+\left(N_{\mathrm{Str}} / N_{R}\right)^{1 / 2} r_{R}
$$

or

$$
r_{\mathrm{Str}, 17} \sim r_{\mathrm{apo}, 17}+0.3 r_{R, 17}^{2} M_{6}^{-1 / 6} T_{4}^{0.1} \zeta_{0.05}^{1 / 2} \Xi_{c, 10}^{1 / 2} .
$$

The column density becomes equal to the Thomson column density $\sigma_{\mathrm{T}}^{-1}$ at

$$
r_{\mathrm{Th}, 17} \sim 0.7 r_{\mathrm{R}, 17}^{2} M_{6}^{-1 / 6} .
$$

The characteristic radii $r_{\mathrm{Str}}, r_{\mathrm{hot}}$, and $r_{\mathrm{apo}}$ are given as a function of $r_{R}$ in Figure 3. Note that in estimating $r_{\text {hot }}$ and $r_{\mathrm{Str}}$ we have ignored absorption and scattering of the incident radiation in the hot part of the tail. In AGN having luminosity $L \leq 10^{46}$ ergs $\mathrm{s}^{-1}$ this is justified since $r_{\mathrm{Th}}$ is larger than $r_{\text {hot }}$ and $r_{\mathrm{Str}}$ in the range where these radii are larger than $r_{\text {apo }}$. In very luminous AGN $\left(L \sim 10^{47}-10^{48}\right.$ ergs s$\left.^{-1}, M_{8} \geq 1\right)$ the column density at $r_{\text {hot }}$ (as defined above)

$$
N\left(r_{\text {hot }}\right)=10^{23} r_{R, 17}^{-4 / 3} L_{44}^{2 / 3}\left(\frac{\xi_{0.3}}{\Xi_{c, 10} T_{\mathrm{C}, 7}}\right)^{2 / 3} \mathrm{~cm}^{-2}
$$

may become larger than $\sigma_{\mathrm{Th}}^{-1}$ at $r_{R} \geq 10^{17} \mathrm{~cm}$, making the effective $r_{\text {hot }} \sim r_{\mathrm{Th}}$. The radiation incident on the remnant is absorbed by cool gas at radii $r$, where

$$
r_{\min }=\max \left[r_{\text {hot }}, r_{\mathrm{apo}}\right] \leq r<r_{\max }=\min \left[r_{\mathrm{Str}}, r_{R}\right],
$$

and reradiated predominantly in broad emission lines (see $\S \S 3.5$ and 4.1).

\subsection{Bound Debris}

Bound debris may play an important role in AGN not only because it provides a source of fuel, but also because it may reprocess a large fraction of the radiation generated near the hole. A full study of the properties of bound debris should include the circularization of this matter and the evolution of the accretion torus. Such an investigation lies beyond the scope of this paper. In this section we will comment briefly on the distribution, density etc., of bound debris using results from the previous sections.

Bound debris has only a small covering fraction $(\sim 0.01$, eq. [3.10]) when it first turns around at $\sim r_{0}$. During the subsequent orbital periods it tends to spread over the available volume within $\sim r_{0}$. The lateral expansion of the debris $\left(v_{\exp } \sim v_{*}\right)$ causes a change in both directions perpendicular to the long axis of the orbit by an amount

$$
\sim 0.1 \mathrm{rad} \text { per orbital period . }
$$

Two general relativistic precession effects may also affect the distribution of bound debris gas: (1) pericenter precession causing a change in the orientation of the orbit (within the orbital plane) of

$$
\sim 1.5 \times \pi r_{\mathrm{S}} / r_{\mathrm{T}} \text { rad per orbital period, }
$$

where $r_{\mathrm{S}}$ and $r_{\mathrm{T}}$ are the Schwarzschild radius of the hole and the tidal disruption radius respectively (eq. [1.1]), and (2) LensThirring precession of the orbital angular momentum around the hole's spin axis. As the matter spirals inward this precession will, in combination with viscous effects, force the matter into the equatorial plane of the hole (Bardeen \& Petterson 1975; Rees 1978). The angular momentum of black holes feeding on gas from randomly distributed stars is expected to be low, but it may be sufficient to determine the orientation of the accretion torus at $r_{\mathrm{T}}$ (Sanders 1984).

Circularization of bound debris may take several orbital periods. It is probably an accelerating process, and the gas will

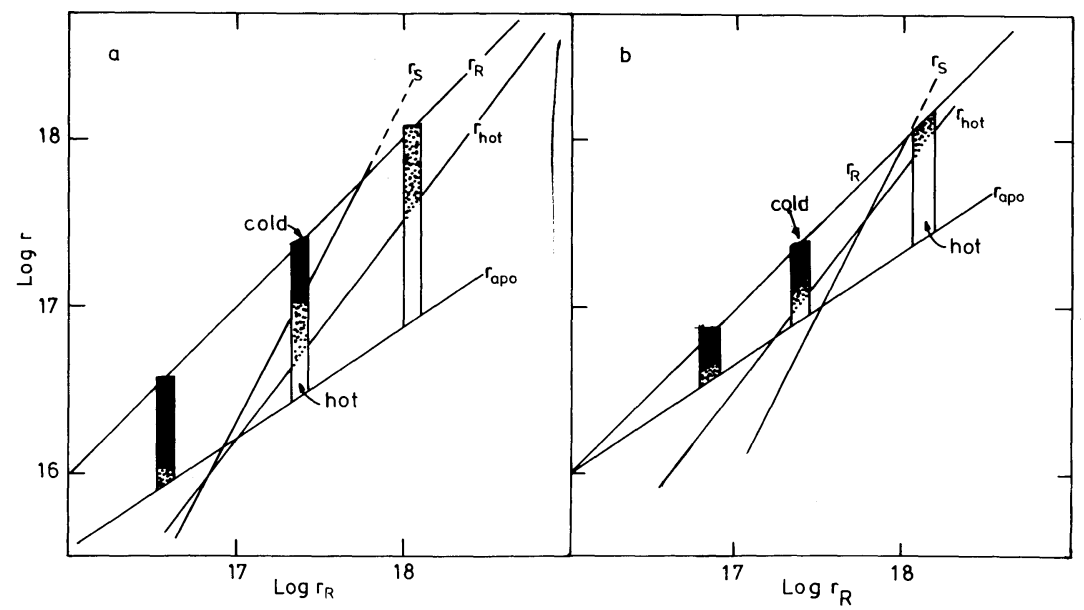

FIG. 3.- Structure of outmoving remnants at different radii from the central source of an AGN with $(a) L_{\text {bol }}=10^{44} \mathrm{ergs} \mathrm{s}^{-1}, M_{\text {hole }}=10^{6} M_{\odot}$ (case a), and (b) $L_{\text {bol }}=10^{46}$ ergs s ${ }^{-1}, M_{\text {hole }}=10^{8} M_{\odot}$ (case b; see $\left.\S 4.1\right)$. The dotted part of the remnant consists of dense, cold cloudlets $\left(T \sim 10^{4} \mathrm{~K}\right)$ embedded in hot gas $\left(T \sim T_{\mathrm{C}} \sim 10^{7-8} \mathrm{~K}\right)$. The mean density and column density along the remnant are given by equations (3.8) and (3.9), respectively. 
therefore spend most of this time at its original apocenter distance. As the matter spreads out within $r_{0}$ the mean density of the bound debris from a single star declines towards $\bar{n}_{\text {single }}\left(r_{0}\right) \sim 1 / 2 M_{\odot} /\left[(4 / 3) \pi r_{0}^{3}\right]$. Conservation of mass for debris moving on Keplerian orbits implies that $\bar{n}_{\text {single }}(r) \propto$ $r^{-3 / 2}$ for $r<r_{0}$, while according to equation (2.2) the mean density of bound debris at radii $r>r_{0}$ decreases as $r^{-4}$. So, ignoring loss of mass and orbital energy, the density of bound gas from a single remnant averaged over the spherical volume within $r$ may be approximated by

$$
\bar{n}_{\text {single }}(r) \approx 10^{8} M_{8}^{-2} \mathrm{~cm}^{-3} \begin{cases}\left(r / r_{0}\right)^{-3 / 2} & \text { for } r<r_{0}, \\ \left(r / r_{0}\right)^{-4} & \text { for } r>r_{0} .\end{cases}
$$

Expanding bound gas will probably also fragment into cold clumps or filaments when the ionization parameter becomes equal to $\Xi_{c}$. It is convenient to write $n_{\text {cold }}$ (eq. [3.17]) in the form

$$
\begin{aligned}
n_{\text {cold }}(r)=3.5 \times 10^{12} \Xi_{c, 10}^{-1} \xi_{0.3} & T_{4}^{-1} M_{8}^{-1 / 3} \\
& \times\left(\frac{L}{L_{\text {Edd }}}\right)\left(\frac{r}{r_{0}}\right)^{-2} \mathrm{~cm}^{-3} .
\end{aligned}
$$

When $\bar{n}_{\text {single }}$ becomes smaller than $n_{\text {cold }} T_{\text {cold }} / T_{\mathrm{C}}$ most of the gas is heated to the Compton temperature. The hydrogen column density of the gas is

$$
\begin{aligned}
N(r) & \sim \bar{n}_{\text {single }}(r) \times r \\
& =1.4 \times 10^{24} M_{8}^{-4 / 3} \mathrm{~cm}^{-2} \begin{cases}\left(r / r_{0}\right)^{-1 / 2} & \text { for } r<r_{0}, \\
\left(r / r_{0}\right)^{-3} & \text { for } r>r_{0} .\end{cases}
\end{aligned}
$$

We may put this equal to the Strömgren column density given in equation (3.26) to estimate the radius where bound debris of a single remnant becomes optically thin to the ionizing radiation from the central source, yielding

$$
r / r_{0} \sim 2.5 M_{8}^{-4 / 9}\left(\zeta_{0.05} \Xi_{c, 10}\right)^{-1 / 3} T_{4}^{-1 / 15} .
$$

These considerations suggest that bound debris close to the central source remains optically thick to the ionizing radiation, but bound debris at larger radii $\left(r \geq r_{0}\right)$ becomes optically thin to the ionizing radiation and heats up to the Compton temperature as it expands into a more spherical volume around the hole.

The mean density (and column density) of bound debris at $r$ decreases on a time scale $x T(r)$ if circularization takes $x$ orbital periods. $T(r)$, the orbital period of debris having apocenter distance $r$, is given by $T(r)=T_{0}\left(r / r_{0}\right)^{3 / 2}$, where $T_{0}$ is given by equation (1.5). We may define a radius $\tilde{r}$ by $v_{\text {tid }} x T(\tilde{r})=1$. Within $\tilde{r}$ the amount of bound debris varies strongly due to the accretion of debris and the appearance of new debris gas. Outside $\tilde{r}$ the density is more constant and is given by equation (3.39) multiplied by $v_{\text {tid }} x T(r) \propto r^{3 / 2}$.

We may estimate the covering fraction of cold, bound debris by assuming that a single remnant breaks up into $\tilde{N}_{\mathrm{cl}} \sim 10^{5}$ (eq. [3.23]) spherical cloudlets with densities given by 3.40 which are distributed spherically symmetrical around the central source. The radius of the clouds is then given by

$$
\begin{aligned}
r_{\mathrm{cl}} \sim 8 \times 10^{12} \Xi_{c, 10}^{1 / 3} \xi_{0.3}^{-1 / 3} \tilde{N}_{5}^{-1 / 3} M_{8}^{1 / 9} T_{4}^{1 / 3} & \\
& \times\left(\frac{L}{L_{\text {Edd }}}\right)^{-1 / 3}\left(\frac{r}{r_{0}}\right)^{2 / 3} \mathrm{~cm},
\end{aligned}
$$

where $\tilde{N}_{5}=\tilde{N}_{\mathrm{cl}} / 10^{5}$. The total covering fraction of all clouds (from more than one remnant) within $r$ is approximately

$$
\begin{aligned}
& \Omega_{\mathrm{total}}(<r) \sim \tilde{N}_{\mathrm{cl}}\left(\frac{r_{\mathrm{cl}}}{4 r}\right)^{2} v_{\text {tid }} x T(r) \\
& \sim 0.1 M_{8}^{-11 / 18} \tilde{N}_{5}^{1 / 3}\left(\frac{\Xi_{c, 10} T_{4}}{\xi_{0.3}}\right)^{2 / 3} \\
& \times\left(\frac{L}{L_{\mathrm{Edd}}}\right)^{-2 / 3}\left(\frac{x}{10}\right)\left(\frac{v_{\mathrm{tid}}}{1 \mathrm{yr}^{-1}}\right)\left(\frac{r}{r_{0}}\right)^{5 / 6}
\end{aligned}
$$

where $r / r_{0}$ should be smaller than $\sim 10$ because at larger radii the clouds become optically thin to the ionizing radiation and the temperature rises to the Compton temperature.

The main conclusion from this section is that a considerable fraction of the central continuum radiation can be reprocessed by cold, optically thick debris gas at $r \leq r_{0}$, in particular if $v_{\text {tid }} x T_{0} \geq 1$.

\subsection{Reprocessing of Radiation by Bound Debris}

Radiation from the central continuum source is reprocessed differently by bound and unbound (cold) debris gas. The densities in unbound remnants at $r>10 r_{0}$ are lower than $\sim 10^{12}$ $\mathrm{cm}^{-3}$. At these densities cold gas reemits most of the received energy in emission lines (see $\S 4.1$ ). At higher densities the line radiation efficiency declines rapidly (Rees, Netzer, \& Ferland 1989; Ferland \& Rees 1988). Dense, bound clouds $\left(n \gg 10^{12} \mathrm{~cm}^{-3}\right.$ ) orbiting within $r \sim r_{0}$ radiate predominantly as blackbodies in thermal equilibrium with the ambient radiation field. The temperature of clouds at a distance $r$ from the central source is

$$
T_{\mathrm{bb}}(r)=1.1 \times 10^{4} L_{45}^{1 / 4} r_{16}^{-1 / 2} \mathrm{~K},
$$

or

$$
T_{\mathrm{bb}}(r)=2.1 \times 10^{4} M_{7}^{-1 / 12}\left(\frac{L}{L_{\mathrm{Edd}}}\right)^{1 / 4}\left(\frac{r}{r_{0}}\right)^{-1 / 2} \mathrm{~K} .
$$

Reprocessing of continuum radiation by such clouds imprints several characteristic absorption (or reflection) and emission features on the spectra of AGN (Guilbert \& Rees 1988; Lightman \& White 1988) such as absorption (or reflection) of X-rays and $\mathrm{Fe} \mathrm{K}$-shell absorption and fluorescence. The energy is reemitted predominantly in the form of thermal emission in the UV (and soft X-ray) regime.

\subsubsection{Absorption of (Soft) $X$-Rays and Low-Frequency Radiation}

The column density of individual clouds, given by

$$
\begin{aligned}
N_{\mathrm{cl}} \sim n_{\mathrm{cold}} r_{\mathrm{cl}} \sim & 10^{25}\left(\frac{\xi_{0.3}}{\Xi_{\mathrm{c}, 10} T_{4}}\right)^{2 / 3} \tilde{N}_{5}^{-1 / 3} \\
& \times\left(\frac{L}{L_{\mathrm{Edd}}}\right)^{2 / 3} M_{8}^{-2 / 9}\left(\frac{r}{r_{0}}\right)^{-4 / 3} \mathrm{~cm}^{-2},
\end{aligned}
$$

is sufficient to absorb the incident radiation (note that $N_{\mathrm{cl}}$ may not exceed the column density given in eq. [3.41]). The X-ray continuum is generally believed to arise at a distance $r_{\mathrm{X}} \sim$ $10 r_{\mathrm{S}} \approx 3 \times 10^{14} M_{8} \mathrm{~cm}$. So, the fraction of the X-ray continuum covered by an individual cloud along our line of sight is

$$
\begin{aligned}
\left(\frac{r_{\mathrm{cl}}}{r_{\mathrm{X}}}\right)^{2} \sim 10^{-3} \Xi_{c, 10}^{2 / 3} \xi_{0.3}^{-2 / 3} \tilde{N}_{5}^{-2 / 3} M_{8}^{-16 / 9} & \\
\times & T_{4}^{2 / 3}\left(\frac{L}{L_{\mathrm{Edd}}}\right)^{-2 / 3}\left(\frac{r}{r_{0}}\right)^{4 / 3} \mathrm{~cm} .
\end{aligned}
$$


The results from the previous section (eq. [3.44]) suggest that total fraction of the X-ray continuum covered by cold clouds, and thus the chance of observing partial absorption by single clouds is appreciable, especially in sources containing small black holes with low values of $L / L_{\mathrm{Edd}}$. In the more powerful sources the absorption is due to many small clouds whereas in weaker sources it is due to a smaller number of clouds covering a larger fraction of the continuum source. We therefore expect soft X-ray absorption in low luminosity sources (Seyferts) to be more important and to exhibit larger variations than in powerful AGN (QSOs). The distribution of bound debris will generally not be spherically symmetric and the amount of absorption may therefore depend on the observer's orientation with respect to the central source.

Free-free absorption of low-frequency radiation by ionized gas surrounding the central source will produce a spectral break at the critical frequency

$$
v_{\text {crit }} \sim 10^{12}\left(\bar{n}_{10} N_{23} T_{5}^{-4 / 3}\right)^{1 / 2} \mathrm{~Hz} .
$$

In our model the column density of the ionized part of the tidal debris is fixed by the critical ionization parameter. Using equation (3.26), we find that free-free absorption by the ionized part of bound debris which is orbiting at $\sim r_{0}$ produces a break at $\sim 10^{12-13} M_{7}^{-1} \mathrm{~Hz}$. Note that this happens to be comparable to the turnover frequency in radio-quiet $\mathrm{AGN}$.

\subsubsection{A Bump in the Ultraviolet}

Dense clouds orbiting between $\sim r_{0}$ and $r_{\mathrm{T}}$ radiate predominantly as black bodies with temperatures between $\sim 10^{4}$ and $\sim 3.10^{5} \mathrm{~K}$ (eq. [3.45]). The distribution of bound matter naturally peaks at $\sim r_{0}$ in sources with a high tidal disruption rate, yielding a thermal emission bump at frequency $v^{*}\left(r_{0}\right) \sim$ $3 k T_{\mathrm{bb}}\left(r_{0}\right) / h \sim 10^{15} \mathrm{~Hz}$. The contribution from hotter clouds at $r<r_{0}$ causes the spectrum to fall off less steeply than the Wien law at $v>v^{*}$. Below $v^{*}$ the spectrum is dominated by cooler cloudlets at $r>r_{0}$.

The combined spectrum of a collection of clouds can be estimated from

$$
F_{v} \propto \int S_{v}(r) r_{\mathrm{cl}}^{2}(r) d \tilde{N}_{\mathrm{cl}}(r)
$$

where $S_{v}(r)$ is the blackbody surface brightness of a single cloud at separation $r$ with temperature given by equation (3.45). Assuming that the number of clouds in the interval $[r, r+d r]$ is given by $d \tilde{N}_{\mathrm{cl}} \propto r^{\alpha} d r$, approximating $S_{v}(r)$ by $S_{v} \propto v^{2} v^{*}(r)$ for $v<v^{*}(v)$ and 0 for $v>v^{*}(r)$, where $v^{*}(r)=3 k T_{\mathrm{bb}}(r) / h$, and integrating over all clouds with $v^{*}>v$, we find using $n_{\mathrm{cl}} \propto r^{-\beta}$ $\left(\beta=3 / 2\right.$ according to eq. [3.40]), and $r_{\mathrm{cl}} \propto n_{\mathrm{cl}}^{-1 / 3}$

$$
F_{v} \propto v^{-(4 \beta / 3)+1-2 \alpha} \sim v^{-1-2 \alpha} .
$$

The nonthermal continuum spectrum in $\mathrm{AGN}$ is often described as a power law with index $\sim-1$. Equation (3.50) illustrates that if the distribution of clouds has a maximum at $r_{0}$ (i.e., if $\alpha$ changes sign at $r_{0}$ ) a thermal UV bump will be produced at $\sim v^{*}\left(r_{0}\right) \sim 10^{15} \mathrm{~Hz}$.

\section{THE BROAD LINE REGION}

\subsection{Broad Emission Lines From Unbound Remnants}

The density of cold gas in the elongated outmoving remnant clouds around the central source is $\leq 10^{12} \mathrm{~cm}^{-3}$ for $L \leq$ $0.1 L_{\text {Edd }}$ (eq. [3.40]). Each remnant contains cold gas with a range of velocities between 0 and $v_{0}$. Photoionization of such clouds by a central source will produce broad emission lines similar to the broad lines observed in AGN. We will now estimate the range of radii, densities and column densities in the broad line region produced by outmoving stellar remnants.

The line luminosity emitted by a single remnant is given by (see Osterbrock 1989)

$$
L_{l}^{\text {single }}=\int_{r_{\min }}^{r_{\max }} j_{l} n_{\mathrm{cl}}^{2}\left(r_{t}\right) \in\left(r_{t}\right) \pi R^{2}\left(r_{R}\right) d r_{t}
$$

where $n_{\mathrm{cl}}\left(r_{t}\right)$ is the density of cold clouds at $r_{t}$ in the remnant tail (eq. [3.17]), $R\left(r_{R}\right)$ is the lateral extent of the remnant ( $R$ is constant along the tail), $\epsilon\left(r_{t}\right)$ is the volume filling factor [equal to $\left.\bar{n}\left(r_{t}\right) / n_{\text {cold }}\left(r_{t}\right)\right]$, and $j_{l}$ is the emission coefficient for a particular line. Note that $r_{\min }$ and $r_{\max }$ both depend on $r_{R}$ (see eq. [3.36]). To a first approximation we may ignore the dependence of the emission coefficient on the temperature and density of the gas. Using results from the previous sections we may approximate the line luminosity given in equation (4.1) by

$$
L_{l}^{\text {single }}\left(r_{\max }\right) \sim \Omega^{\text {single }}\left(r_{\max }\right)\left(\frac{\xi L_{\text {bol }}}{h v_{R}}\right)\left(\frac{j_{l}}{\alpha}\right)\left(\frac{N\left(r_{\max }\right)}{N_{\text {Str }}}\right),
$$

where

$$
\Omega^{\text {single }}\left(r_{\max }\right)=\left(\frac{r_{\text {max }}}{r_{R}}\right)^{-2} \Omega\left(r_{R}\right)
$$

and $\Omega\left(r_{R}\right)$ is given in equation (3.10). At small $r_{R}$ most line radiation comes from $r_{\max } \approx r_{\min }$, whereas at larger radii the line radiation comes from around $r_{\mathrm{Str}}$ or $r_{R}$.

For a constant tidal disruption rate the number of unbound remnants in the interval $\left[r_{R}, r_{R}+d r_{R}\right]$ is

$$
\frac{v_{\mathrm{tid}}}{v_{0}} d r_{R} \approx 3\left(\frac{v_{\mathrm{tid}}}{1 \mathrm{yr}^{-1}}\right) M_{8}^{-1 / 6} d r_{R, 17}
$$

The line luminosity emitted in the interval $[r, r+d r]$, where $r=r_{\max }\left(r_{R}\right)$, by remnants with $r_{R}$ in the interval $\left[r_{R}, r_{R}+d r_{R}\right]$, is given by

$$
d L_{l}(r)=L_{l}^{\text {single }}(r) \frac{v_{\text {tid }}}{v_{0}} d r_{R}
$$

which defines the distribution of the line luminosity emitted by outflowing remnants in AGN.

In Figure 4 the line luminosity integrated out to radius $r$ is given for two cases. In case (a) $\left(L_{\text {bol }}=10^{44} \mathrm{ergs} \mathrm{s}^{-1}, M_{6}=1\right)$ most line emission comes from the tip of the tail, at $\sim r_{\text {apo }}$, at small radii, and from $\sim r_{\mathrm{Str}}>r_{\text {apo }}$ at larger $r_{R}$. The transition occurs at

$$
r_{17}^{*}=0.7 M_{8}^{5 / 12} T_{\text {cold, } 4}^{-0.05},
$$

where we have omitted factors of order unity. Another change of slope occurs when $r_{\mathrm{Str}}=r_{R}$, at

$$
r_{17}^{* *}=11 M_{8}^{1 / 6} T_{\text {cold, } 4}^{-0.1} \text {. }
$$

The luminosity distribution then has the form

$$
\frac{d}{d r} L_{l}(r) \propto \begin{cases}r^{3 / 2} & \text { for } r<r^{*} \\ r^{-3 / 2} & \text { for } r^{*}<r<r^{* *} \\ r^{-2} & \text { for } r>r^{* *}\end{cases}
$$

About half of the total line emission comes from $r<r^{*}$, and 


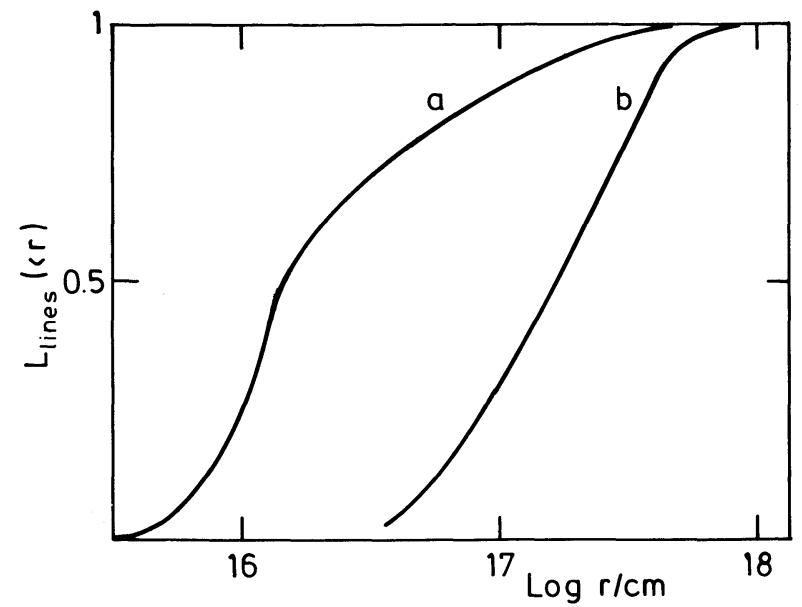

Fig. 4--Integrated line luminosity normalized to the total line luminosity emitted by cold gas from outmoving remnants in a Seyfert type (case a) and a QSO type (case b) AGN. In case a we find that the BLR extends from $\sim 10^{16}$ to $\sim 10^{17} \mathrm{~cm}$, with typical densities and column densities of $10^{8.5-10.5} \mathrm{~cm}^{-3}$ and $10^{25-27} \mathrm{~cm}^{-2}$, respectively. In case (b) we find $r_{\text {BLR }} \sim 10^{17-17.5} \mathrm{~cm}$ with densities $\sim 10^{9.5-11} \mathrm{~cm}^{-3}$ and column densities $\sim 10^{25-26} \mathrm{~cm}^{-2}$.

the other half from $r^{*}<r<r^{* *}$. The range of densities and column densities in the line emitting region are

$$
\left\{\begin{array}{l}
r \sim 10^{16}-10^{17} \mathrm{~cm} \\
n_{\text {cold }} \sim 10^{11}-10^{9} \mathrm{~cm}^{-3} \\
N_{\mathrm{H}} \sim 10^{27}-10^{25} \mathrm{~cm}^{-2}
\end{array} .\right.
$$

In case (b) we have an extra region where most line radiation comes from just beyond $r_{\text {hot }}$. The transitions between the different regions are now determined by

$$
\begin{gathered}
r_{\text {apo }}=r_{\text {hot }} \text { at } r_{17}^{+}=0.6 M_{8}^{11 / 18} L_{46}^{-1 / 3} T_{\mathrm{C}, 7}^{1 / 3}, \\
r_{\text {hot }}=r_{\text {Str }} \text { at } r_{17}^{++}=5 T_{\text {cold, } 4}^{0.2} T_{\mathrm{C}, 7}^{1 / 3} L_{46}^{-1 / 3} M_{8}^{-1 / 6},
\end{gathered}
$$

and $r_{\mathrm{Str}}=r_{R}$ at $r^{* *}$ given by equation (4.7). The line luminosity distribution is now given by

$$
\frac{d}{d r} L_{l}(r) \propto \begin{cases}r^{3 / 2} & \text { for } r<r^{+}, \\ r^{-3 / 4} & \text { for } r^{+}<r<r^{++}, \\ r^{-3 / 2} & \text { for } r^{++}<r<r^{* *}, \\ r^{-2} & \text { for } r>r^{* *} .\end{cases}
$$

The integrated line luminosity is again given in Figure 4. About $10 \%$ comes from $r<r^{+}$; another $10 \%$ from $r>r^{++}$. The largest contribution comes from the region $r^{+}<r<r^{++}$. In this case the line emitting region exhibits the following range in densities and column densities

$$
\left\{\begin{array}{l}
r \sim 10^{16.5}-10^{17.4} \mathrm{~cm}, \\
n_{\text {cold }} \sim 10^{11.5}-10^{9.5} \mathrm{~cm}^{-3} \\
N_{\mathrm{H}} \sim 10^{26.5}-10^{24.5} \mathrm{~cm}^{-2}
\end{array} .\right.
$$

In this case line emission occurs at larger radii because a large part of the tails is heated to the Compton temperature.

The ratio of the luminosity emitted in the lines to the luminosity of the central continuum source is determined by the covering fraction of the line emitting parts of outmoving remnants, which is given by

$$
\Omega_{l}(r)=\int_{0}^{r} \frac{v_{\mathrm{tid}}}{v_{0}} \Omega^{\text {single }}\left(r_{\max }\right) d r_{R} .
$$

Evaluating the integral in case (a) for $r=+\infty$ yields

$$
\Omega_{l} \sim 0.15\left(\frac{v_{\text {tid }}}{1 \mathrm{yr}^{-1}}\right) M_{8}^{-11 / 24} T_{\text {cold }, 4}^{-1 / 8} \sim 1\left(\frac{v_{\text {tid }}}{1 \mathrm{yr}^{-1}}\right) .
$$

For case (b) we find

$$
\Omega_{l} \sim 0.15\left(\frac{v_{\mathrm{tid}}}{1 \mathrm{yr}^{-1}}\right) M_{8}^{-1 / 4} L_{46}^{-5 / 6} T_{\mathrm{C}, 7}^{5 / 6} \sim 0.15\left(\frac{v_{\mathrm{tid}}}{1 \mathrm{yr}^{-1}}\right) .
$$

The scaling with $M$ and $L$ given above is only valid for limited ranges around the chosen values of $M$ and $L$. It is possible to obtain a lower limit to the amount of continuum radiation that is reprocessed by unbound stellar remnants by calculating the covering fraction of the optically thick part of the remnants. The distance along the remnant tail where the electron scattering optical depth becomes 1 is given by equation (3.34). The total covering fraction is then

$$
\begin{aligned}
\Omega_{\mathrm{Th}}= & \int \frac{v_{\mathrm{tid}}}{v_{0}}\left(\frac{\max \left[r_{\mathrm{apo}}, r_{\mathrm{Th}}\right]}{r_{R}}\right)^{-2} \Omega_{R} d r_{R} \\
& \sim 0.05\left(\frac{v_{\mathrm{tid}}}{1 \mathrm{yr}^{-1}}\right) M_{8}^{-11 / 24}
\end{aligned}
$$

Let us summarize the main results from this section. A considerable fraction of the central continuum radiation in AGN with a tidal disruption rate $\geq 0.1 \mathrm{yr}^{-1}$ is intercepted by optically thick, radially elongated remnants at separations $10^{16}$ $10^{18} \mathrm{~cm}$ from the central source. The unbound remnants contain cold gas with densities which are generally lower than $\sim 10^{12} \mathrm{~cm}^{-2}$ (see eq. [3.40]) and reemit the received energy predominantly in Doppler broadened lines with widths of several thousand $\mathrm{km} \mathrm{s}^{-1}$. Matter beyond the Strömgren radius is heated by hard X-rays and may be important as the source of low ionization lines such as $\mathrm{Mg}$ II and the Balmer lines. As the remnants move out and the density and column density decline, more matter goes into the hot phase, and the remnant becomes optically thin to the ionizing radiation from the central source. As a result, the line emission is confined to a rather well-defined region within the range $10^{16}-10^{18} \mathrm{~cm}$, characterized by densities (of cold gas) of $\sim 10^{12}$ to $\sim 10^{9}$ $\mathrm{cm}^{-3}$ and column densities of $\sim 10^{27}$ to $\sim 10^{24} \mathrm{~cm}^{-2}$ (cases a and b). Forbidden lines such as [O III] will at most have very weak broad components.

The number of remnants contributing to the line is only $\sim 30\left(v_{\text {tid }} / 1 \mathrm{yr}^{-1}\right)$. A variety of broad line profiles may be produced by such a small number of remnants. The mean line profile in a sample of AGN will be logarithmic-like, but broad line profiles in individual sources may have bumps, shoulders, and asymmetries (Roos \& Espey 1992, in preparation).

\subsection{Line Emission from Bound Debris}

Cold debris gas at separations $\leq r_{0}$ is characterized by high densities (greater than $10^{12} \mathrm{~cm}^{-3}, \Xi \sim 10$ ) and high column densities. Such clouds will cool predominantly by emitting radiation along with a few prominent spectral lines such as $\mathrm{Fe}$, $\mathrm{K} \alpha$, and Ly $\alpha$ (Ferland \& Rees 1988). Line emission becomes the dominant cooling mechanism at lower densities (larger separations). It seems therefore likely that unbound debris gas emits more energy in lines than bound debris gas. The relative contribution of bound debris to the broad lines depend on various uncertain factors such as the amount and distribution of bound debris and the luminosity of the central source. A few differences between bound and unbound debris may be noted. 
Bound debris has higher densities and will emit stronger Balmer continua and more Ly $\alpha$. It is ionized at radii larger than $\sim 10 r_{0}$ (eq. [3.42]). It moves radially inward and outward with characteristic velocity $\sim v_{0}$ yielding symmetric logarithmic-like profiles (Shields 1989). The lines may be broader than the lines emitted by unbound debris because unbound debris has a maximum velocity $v_{0}$. Its characteristic distance to the central continuum source is $\sim r_{0}$, yielding a dynamical time scale of $\sim t_{0} \sim 190 M_{8}^{1 / 2}$ days and a characteristic time scale for response to variations in the ionizing continuum $\sim r_{0} / c \sim 4.6 M_{8}^{2 / 3}$ day. The high-velocity wings of the lines emitted by bound debris respond faster than the cores, whereas in the case of outmoving remnants, the cores and the blue wings of the lines are expected to respond faster than the red wings.

\subsection{Comparison with the Observations}

\subsubsection{Form and Width of the Lines}

The broad permitted lines in the spectra of Seyferts and QSOs have characteristic widths of several thousand $\mathrm{km} \mathrm{s}^{-1}$ in weak sources to $\sim 10^{4} \mathrm{~km} \mathrm{~s}^{-1}$ in bright QSOs. Joly et al. (1985) find that the linewidths are approximately proportional to $L^{0.2 \pm 0.04}$. The fact that the tidal disruption model reproduces the typical width and its weak dependence on luminosity (linewidth $\propto L^{1 / 6}$, for constant $L / L_{\text {Edd }}$ ) is a strong argument in favor of it (Shields 1989).

The predominantly radial motions of the tidal debris gas are well-suited to reproduce the basic form of the broad lines, which is often described as logarithmic (e.g., Capriotti, Foltz, \& Byard 1980). This basic form should be regarded as a very crude mean, because broad permitted lines show a wide variety of profile shapes from very peaked symmetric to almost flattopped and often asymmetric and bumpy profiles (e.g., van Groningen 1983; Stirpe 1990). Osterbrock \& Shuder (1982; see also Rafanelli 1985) find that the Balmer lines are often asymmetric with the blue and red intensities (at half-maximum) differing sometimes by $\sim 40 \%$. In the tidal debris model the differences in profile shapes among otherwise similar AGN is due to the small number of tidal remnants which contribute to the broad line. The lines emitted by outmoving remnants are calculated in models (Roos \& Espey 1992, in preparation) exhibit a variety of profile shapes similar to the observed lines. The contribution from bound debris is expected to be more symmetric (Shields 1989). The broad lines formed by outmoving remnants become more symmetric as the number of remnants contributing to the lines increase (with increasing tidal disruption rate and with increasing distance to the central source).

\subsubsection{Photoionization Models}

It is generally accepted that the broad (as well as the narrow) lines originate from cold $\left(T \sim 10^{4} \mathrm{~K}\right)$ gas which is photoionized by the central continuum source (e.g., Osterbrock, 1985). The early photoionization models were moderately successful in explaining the ratios of the broad lines in AGN employing the following ranges in density, column density and ionization parameter for the broad line gas: $10^{9}<n<10^{10} \mathrm{~cm}^{-3}, N_{\mathrm{H}}>$ $2 \times 10^{22} \mathrm{~cm}^{-2}$ and $0.01<U<0.1$ (Davidson \& Netzer 1979; Kwan \& Krolik 1981; Ferland \& Netzer 1984). The size of the broad line region (BLR) can be deduced from the product $U n$ :

$$
r_{\mathrm{BLR}} \sim 3.5 \times 10^{17}\left(\frac{U n}{10^{10} \mathrm{~cm}^{-3}}\right)^{-1 / 2} L_{46, \mathrm{ion}}^{1 / 2}\left(\frac{\langle h v\rangle}{1 \mathrm{Ry}}\right)^{-1 / 2} \mathrm{~cm},
$$

where $L_{46 \text {,ion }}$ is the ionizing luminosity and $\langle h v\rangle$ is the mean ionizing photon energy. It is interesting to compare the gas velocity inferred from the linewidths with the Kepler velocity of gas moving at a distance $r_{\text {BLR }}$ from the central black hole given by

$$
V_{\text {Kepler }}=1950\left(\frac{r_{\text {BLR }}}{3.5 \times 10^{17} \mathrm{~cm}}\right)^{-1 / 2} M_{8}^{1 / 2} \mathrm{~km} \mathrm{~s}^{-1} .
$$

Estimates of the black hole masses based on the assumption that the broad line gas moves on Keplerian orbits yield unrealistically high values of $10^{10}-10^{12} M_{\odot}$ for bright QSOs (Wandel \& Yahil 1985; Joly 1987) if $U n \sim 10^{8-9} \mathrm{~cm}^{-3}$ as in the early photoionization models.

Within the last few years this simple photoionization model sometimes referred to as the "standard model," has been confronted with some major difficulties, reviewed by Netzer (1989). Firstly, Netzer (1985) and Collin-Souffrin (1986) pointed out that the standard model cannot account for the strengths of the low ionization lines (LILs) like the Balmer lines, $\mathrm{Mg}$ II, Ca II, and $\mathrm{Fe}$ II and the ratio of the energy emitted in these lines to the energy emitted by the high ionization lines (HILs) such as Ly $\alpha, N$ v, C IV, He II, etc. This comprises the "energy budget problem" and the "Fe II problem." It was therefore argued by Collin-Souffrin et al. (1986) that at least two physically different line emitting regions exist in the BLR: the HIL region and the LIL region (Collin-Souffrin et al. 1986). The conditions in the HIL emitting gas are similar to those of the standard model while the LILs are emitted by cool denser gas which is shielded from the ionizing UV source and is heated by harder (predominantly $\mathrm{X}$-ray) radiation and/or by a nonradiative mechanism. The physical separation between the HIL and LIL emitting gas is supported by the observations that the HILs are systematically blueshifted with respect to the LILs (Gaskell 1982; Wilkes 1984). Secondly, the rapid response of the broad lines to variations in the luminosity of the continuum source indicates that the radius of the BLR estimated from equation (4.16) using the parameters of the standard model is too large by at least one order of magnitude (see Peterson 1988 for a review). The size of the broad line region is now estimated to be $\sim 10^{16-17} \mathrm{~cm}$ in Seyferts and $\sim 10^{17-18} \mathrm{~cm}$ in QSOs, implying that $U n \sim 10^{9-11} \mathrm{~cm}^{-3}$ instead of $\sim 10^{8} \mathrm{~cm}^{-3}$ (see also Padovani et al. 1990).

These problems have prompted new photoionization studies covering regions in parameter space having higher densities and/or ionization parameters, and higher column densities (e.g., Rees et al. 1989; Ferland \& Persson 1989). Rees et al. (1989) have investigated models having a range of densities and radii. One of their results is that the density distribution in the BLR must have a sharp cutoff at $\sim 10^{8} \mathrm{~cm}^{-3}$ in order to prevent too much broad [O III]. They do not solve the line ratio problems mentioned above. Ferland \& Persson (1989) investigated models with high column density and high ionization parameter $\left(N_{\mathrm{H}}>10^{25} \mathrm{~cm}^{-2}\right.$ and $\left.U=0.3\right)$. They were remarkably successful in reproducing all of the observed line ratios in the BLR.

The tidal disruption model naturally explains the typical radii and densities of the BLR. The whole BLR extends over a range in radii and densities which is not much larger than a factor $\sim 30$ and $10^{3}$, respectively, which solves the problem of the lack of broad [O III]. The high column densities and ionization parameter $(\Xi \sim 10$ and $U \sim 1)$ predicted by the model are very similar to the parameters found by Ferland $\&$ Persson (1989) to reproduce the observed line ratios. Large expanding 
clouds having high column density are attractive not only because they produce strong low ionization lines, but also because they do not require a confining medium.

High ionization lines are emitted by the tails of the remnants pointing toward the central source and perhaps also by bound debris. Low ionization lines are produced further out along the remnant in the predominantly neutral zone, which is shielded from the central optical/UV radiation and heated by hard $\mathrm{X}$-rays from the central source. HILs are thus expected to respond faster to variations in the continuum luminosity than LILs. This is indeed found to be the case in the recent extensive monitoring program on NGC 5548 (Peterson et al. 1990; Clavel et al. 1990), where $\operatorname{Ly} \alpha$ and C IV were found to lag behind the continuum by about 8 days, whereas for the Balmer lines the lag was about 20-25 days.

In photoionization structure and radiation transfer in an infinite slab of constant density and velocity, which is oriented perpendicular to the direction of the source of ionizing radiation. The results of such calculations do not directly apply to the outmoving remnants in the tidal disruption model. The density and velocity in these clouds increase outward along the remnant (also the cold gas is clumpy). The velocity shear will affect the line radiation transfer, and the optical depth for the line radiation generated in the remnant depends both on radial position along the remnant and direction with respect to the velocity vector of the remnant. The optical depth may be smallest for photons emitted by the tail and traveling in the tangential (and backward) direction. The column density encountered by photons traveling from the tail in the tangential direction can be a factor 10-100 smaller than in the radial direction. Such differences may help to explain the differences between high and low ionization lines such as the blueshift of the HILs with respect to the LILs, and the symmetry of the Ly $\alpha$ line.

\subsubsection{Geometry and Kinematics of the Broad Line Region}

Two of the major questions regarding the kinematics of the BLR are these: is the gas bound or unbound to the central hole, and, is the motion predominantly radial (outflow or inflow), circular or chaotic? The tidal debris model suggests that the inner part of the BLR may be bound while the outer part is moving radially outward. It was noted above that the mass of the central object derived from the standard photoionization model and the assumption of Keplerian motion appears to be too large by about two orders of magnitude. The discrepancy is probably partly due to the low value of $U n \sim 10^{8} \mathrm{~cm}^{-3}$ in the standard model. Using instead $U n \sim 10^{10} \mathrm{~cm}^{-3}$ still leaves us with a discrepancy of about one order of magnitude suggesting that the gas is not bound to the hole. There are other indications as well that there is outflow of cold gas from AGN. First, the broad wings of the narrow lines that have been found in some AGN (and the width of the narrow lines themselves) indicate non-Keplerian motion at distances $\geq 1 \mathrm{pc}$ from the nucleus. Second, the spectra of about $10 \%$ of all QSOs have broad absorption troughs on the blue side of the high ionization lines indicating outflow with velocities up to $0.1 c$ at radii $\geq 1 \mathrm{pc}$. The properties of the broad absorption systems are correlated with those of the broad lines in these QSOs (see $\S 5$ ). Third, line variability studies of QSOs show that often the cores of broad emission lines vary more than the wings, which is opposite to what one would expect for Keplerian motions (Gondhalekar, O'Brien, \& Wilson 1986; O'Brien, Zheng, \& Wilson 1989). Such behavior is expected if the broad lines are emitted by outmoving remnants since the velocity increases outward along the remnants. The cores and the blue wings are then expected to respond faster to continuum variations than the red wings. The results of the variability studies of QSOs do not agree with the results of the recent monitoring campaigns of NGC 4151 and NGC 5548 which show that the wings of the HILs respond faster to continuum variations than the core. This is inconsistent with pure outflow (or pure inflow) models and it suggests that the HILs are, at least partly, emitted by bound matter.

Another major question regarding the structure of the BLR is: does it have a spherically symmetric (perhaps patchy) distribution or is it in a flattened, perhaps axially symmetric configuration? Accretion disks are often invoked to explain the properties of BLR (Collin-Souffrin et al. 1988) as well as the big blue bump (BBB; Shields 1978). A strong argument in favor of a disklike geometry is the low level of X-ray (and Lyman edge) absorption in combination with a large covering fraction of the broad line emitting gas. Note that this type of model implies that most of the disk surface is not illuminated directly by the central source. Another piece of evidence is the anticorrelation between the width of the broad $\mathrm{H} \beta$ line and the ratio $R$ of the radio core to the radio lobe emission found by Wills $\&$ Browne (1986) for a sample of radio-loud QSOs and broad line radio galaxies. The observed correlation would be expected if $R$ is determined primarily by orientation, in particular Doppler boosting of a relativistic jet, and the broad lines are emitted by gas having some axially symmetric distribution with respect to the jet. However, the observational evidence in favor of large accretion disks in AGN remains rather scarce and several properties of the BLR (and of the BBB; see Antonucci 1987) seem to be in conflict with this model. The characteristic symmetric double-horned line profile predicted by the simple disk models is never observed. Double-horned profiles are observed in a few cases, but the horns are asymmetrically placed with respect to the line center and have different strengths. In fact they look more like extreme examples of the humps or shoulders that are often observed in broad line profiles. The asymmetric double-horned line profile in Arp 102B has been modeled by a relativistic accretion disk (Chen, Halpern, \& Filippenko 1989), but Miller \& Peterson (1990) have recently shown that this model is inconsistent with observed profile variations. The form of the broad lines as well as their response to changes in the ionizing continuum suggest a more complicated, multicomponent structure of the BLR which is dominated by in- or outflow or perhaps chaotic motions. The tidal disruption model seems well suited to explain the form of the lines, but a serious problem remains: why are broad line clouds not seen in absorption? The stellar remnants producing the broad line region are large enough to cover the central continuum source in UV and soft X-ray. Perhaps the nucleus would not be classified as an AGN when our line of sight happens to pass through an optically thick remnant clouds. Note that optically thin remnants moving outside the BLR cover a large fraction of the BLR and may produce broad absorption systems similar to those in BAL QSOs (see $\S 5.3$ ).

\subsubsection{Variability}

Spectroscopic variability observations are a prime tool for unraveling the kinematics and geometry of spectral components of AGN such as the BLR (e.g., Blandford \& McKee 1982; Capriotti et al. 1982). We have mentioned already that reverberation studies of some AGN (in particular NGC 5548; Clavel et al. 1990) have revealed that the size of the BLR in 
these sources is considerably smaller than previously believed. X-ray observations of NGC 5548 (Kaastra \& Barr 1989) indicate that it contains a black hole of about $10^{7} M_{\odot}$. The estimated bolometric luminosity is $\sim 10^{44} \mathrm{ergs} \mathrm{s}^{-1}$, so it should be comparable to case (a) (Fig. 4). The size of the BLR predicted in the tidal disruption model is consistent with the results of the monitoring campaign. Note however that these indicate that bound matter should also contribute to the broad lines, in particular to the HILs. In- and outflow are more or less balanced for bound debris at $r \leq 10 r_{0}$. The red and blue wings of the broad lines are therefore expected to respond simultaneously and before the core of the line if broad lines are produced by bound debris. The core of the line and the blue wing will respond faster than the red wing if broad lines are predominantly due to outmoving remnants. Note, however that this systematic effect may be overshadowed by the intrinsic irregularity of the distribution of stellar debris at small radii.

The tidal disruption model predicts that the inner part of the broad line region composed of only a few outmoving remnants is more irregular than the outer part. This implies that the line profile changes in response to continuum variations are more irregular than the full line profile. Variability studies do indeed seem to accentuate the multicomponent nature of the BLR (e.g., Peterson 1988). The line profiles in the difference spectra of NGC 5548 obtained by Stirpe et al. (1988; see also Stirpe 1989) for example look more irregular than the line profiles in the original spectra (see also the results on Ak 120 of Alloin et al. 1986).

The whole broad line region has to be replenished in $\sim 30 \mathrm{yr}$ if it is not bound to the hole. In the tidal disruption model the injection of new broad line clouds is a stochastic process with a mean frequency $v_{\text {tid }}$. The appearance of new bound debris from a disrupted star on a characteristic time scale $T_{0}$ is accompanied by, or followed by, an increase in the energy output, perhaps in the form of one or more flares. Such an event may already have been observed in NGC 5548 by Peterson \& Ferland (1986, hereafter P\&F). Optical spectra taken 3 months apart showed an increase in the optical continuum by about $70 \%$ accompanied by the appearance of a strong and very broad He II $4686 \AA$ emission line, while the Balmer lines did not change appreciably. The mass of the gas emitting this line, estimated from the emission measure, is $\sim 0.8 n_{10}^{-1} M_{\odot}$. Different interpretations of this event have been discussed by Kallman \& Elitzur (1988) such as (1) a large increase in the ionization parameter of clouds with covering fraction $\sim 1$, (2) a flare in the extreme UV continuum, and (3) an increase in the cloud covering fraction (to $\sim 1$ ) of clouds with low column density $\left(\sim 10^{22-23} \mathrm{~cm}^{-2}\right)$. The last interpretation was favored by $P \& F$ who suggested that a new, highly ionized gas component, perhaps debris form a disrupted star, has entered the BLR. If this interpretation is correct it seems most likely that we are dealing with bound debris which has just turned around at $r_{0}\left(\sim 3 \times 10^{15} \mathrm{~cm}\right.$ for a $10^{7}$ solar mass black hole $)$ and begun orbiting the central hole. This would explain the appearance of highly ionized gas with a large covering fraction on a time scale of $r_{0} / v_{0} \sim$ one or two months. Note that the time scale for the appearance of the gas indicates that it lies inside the BLR. If the inner radius of the BLR is about 8 light days, as suggested by the results of the recent monitoring campaign on NGC 5548 , the time scale for appearance would be about $8 c / v_{0} \sim$ 500 days. The broad $\mathrm{He}$ line could not be produced by the unbound outmoving part of a debris cloud, because that would have a covering fraction of only about 0.01 and it would not produce a very broad and symmetric line. The mean density and column density of the bound part of a debris cloud would be $\sim 10^{10} \mathrm{~cm}^{-3}$ and $\sim 3 \times 10^{25} \mathrm{~cm}^{-2}$, respectively if it were spread out uniformly within $r_{0}$. The lines produced by this gas would also be broader than the lines produced by outmoving remnants at $r>3 \times 10^{16} \mathrm{~cm}$. Detailed photoionization calculations of high-density, high column density, and high ionization parameter gas are required to decide whether the $\mathrm{He}$ $\mathrm{II} /(\mathrm{H} \alpha$ or $\mathrm{H} \beta)$ ratio is consistent with observations. Also, strong $\mathrm{X}$-ray absorption and reradiation of the intercepted energy in the UV (continuum and lines) are expected. There is evidence that events such as the one observed by P\&F have occurred more often in NGC 5548 (van Groningen 1991, private communication). Intensive multiwavelength monitoring of AGN during such events, over a period longer than 3 months, will undoubtedly yield new and essential clues to the broad line puzzle.

\section{DISCUSSION}

The tidal debris model discussed in the previous sections predicts a number of characteristic properties of cold gas over a large range of radii in AGN. The model is particularly successful in explaining the size, densities, column densities, and velocities of the BLR (as discussed in the previous section), but it may also help to understand some other features in AGN spectra such as the big blue bump, X-ray absorption and emission features, broad absorption lines, the narrow line region and absorption of low-frequency radiation. In this section we will briefly discuss these features leaving a more extensive investigation to future work.

\subsection{X-Ray Absorption and Emission}

The X-ray satellites EXOSAT and Ginga have already provided many interesting results on the spectra of AGN in the range $0.1-10 \mathrm{keV}$. The high-energy part of the spectrum (1-10 $\mathrm{keV}$ ) is generally well described by a power law with index $\sim 0.7$. In the lower energy range the spectra are often more complex showing partial, and sometimes variable absorption, reflection and fluorescence by cold gas with column densities $\sim 10^{21}-10^{23} \mathrm{~cm}^{-2}$, and a very steep soft X-ray excess dominating below $\sim 0.1-1 \mathrm{keV}$ (e.g., Turner \& Pounds 1989). Both the hard and soft $\mathrm{X}$-rays are thought to originate from a region $\sim 5-10$ Schwarzschild radii from an accreting black hole. This is supported by the very rapid variability, down to a few hundred seconds in the weaker sources, of these components (e.g., McHardy 1989) and by the correlation between them. It was pointed out by Guilbert \& Rees (1988, hereafter G\&R) and Lightman \& White $(1988$, hereafter L\&W) that several of the $\mathrm{X}$-ray properties described above are expected as a result of reprocessing of the hard nonthermal radiation from the central source by cold dense matter around it. Such matter is generally expected to be present in accretion driven AGN either in an accretion disk or in some other perhaps more spherically symmetric configuration. The tidal debris model predicts that part of the gas near the hole is in a relatively small accretion torus at $r_{\mathrm{T}}$ (eq. [1.1]) which is surrounded by a patchy, perhaps more spherically symmetric, distribution of gas clouds and filaments having high column densities and $\Xi \sim 10$. Blackbody radiation from dense gas near $r_{\mathrm{T}}$ (temperature $\geq 10^{5} \mathrm{~K}$ ) may be responsible for the soft X-ray excess. Combined $I U E$ and EXOSAT observations of some AGN suggests that the soft $\mathrm{X}$-ray excess may be modeled by the high energy tail of a blackbody spectrum with a temperature of a few times $10^{5} \mathrm{~K}$ 
(Arnaud et al. 1985). In the case of Mkn 841 the luminosity of $\sim 2.10^{46} \mathrm{ergs} \mathrm{s}^{-1}$ and temperature of $2.10^{5} \mathrm{~K}$ imply a radius of the emitting region of $\sim 3.10^{14} \mathrm{~cm}$, which is consistent with $\sim 10 r_{\mathrm{s}}$ if the luminosity is equal to the Eddington luminosity.

Cold debris gas having large column densities will produce high-energy cutoffs due to photoelectric absorption. Column densities of $\sim 10^{21}-10^{23} \mathrm{~cm}^{-2}$ and large covering fractions $(\geq 0.5)$ are commonly found in low-luminosity AGN $\left(L_{\mathbf{X}}<\right.$ $10^{44} \mathrm{ergs}^{-1}$ ), but rarely in AGN with higher X-ray luminosities (Petre et al. 1984; Reichert et al. 1983). The present X-ray satellites cannot detect absorption cutoffs above $10 \mathrm{keV}$ due to intervening gas with column densities greater than $10^{25} \mathrm{~cm}^{-5}$. Note however that Ginga observations of Seyfert galaxies (e.g., Morisawa et al. 1990) have found evidence for absorption by cold matter having column densities of order $10^{24}-10^{25} \mathrm{~cm}^{-2}$, covering about half the central source. Further evidence that high column density, cold gas is indeed present near the central energy source is provided by the discovery of $\mathrm{Fe} \mathrm{K}$-emission and absorption features in several AGN (e.g., Nandra et al. 1989; Pounds et al. 1989), indicating large column densities $\geq 5.10^{23} \mathrm{~cm}^{-2}$ covering a large fraction of the central source. The ionization parameter of the gas can be estimated from the position of the Fe K-edge. Pounds et al. (1989) infer an ionization parameter $\Xi \sim 10$ for the absorbing gas consistent with the tidal debris model.

\subsection{The Big Blue Bump}

In the optical-UV most AGN exhibit an excess above the overall $(\alpha \sim-1)$ power law characterizing the continuum spectrum from the infrared to the hard X-ray regime. This flat component $(\alpha \sim-0.5)$, often referred to as the big blue bump (BBB), extends from $\sim 10^{14.5} \mathrm{~Hz}$ into the unexplored EUV gap ( $\sim 912 \AA-0.2 \mathrm{keV}$ ) where it must roll-over to connect with the soft X-ray part of the spectrum (e.g., Sanders et al. 1989, and references therein). IUE observations of high-redshift QSOs indicate that in these objects the flat $(\alpha \sim 0.5) \mathrm{UV}$ continuum may extend to $\leq 600 \AA$ (e.g., Reimers et al. 1989), but Seyferts may have a cooler bump (Clavel, Wamsteker, \& Glass 1989). Many authors favor a thermal origin for the bump, either thermal emission from a geometrically thin, optically thick viscous accretion disk (Shields 1978; Edelson \& Malkan 1986, and references therein), or radiation emitted by dense, cold matter which is irradiated by the central source (G\&R, L\&W). QSO continua in the optical/UV can be fitted with singletemperature blackbody spectra with temperatures in the narrow range $2.10^{4}-3.10^{4} \mathrm{~K}$ (Malkan 1983). Note however, that a wider range of temperatures from $10^{4} \mathrm{~K}$ to $\geq 10^{5} \mathrm{~K}$ may be present, especially if the steep soft X-ray excess found in several AGN represents the high-energy extension of the bump (Pounds et al. 1986; Czerny \& Elvis 1987). The effective size of the region emitting the BBB can be estimated, assuming it has a spherical geometry and radiates like a blackbody, from

$$
R_{\mathrm{UV}}=8.3 \times 10^{15} L_{\mathrm{UV}, 46}^{1 / 2}\left(\frac{T}{3.10^{4} \mathrm{~K}}\right)^{-2} \mathrm{~cm}
$$

Variability studies indicate sizes smaller than $\sim 10^{17} \mathrm{~cm}$ (Cutri et al. 1985).

Bound debris heated by the central source is expected to emit an optical-UV bump similar to the observed BBB (see $\S 3.5 .2)$. A large fraction of the radiation from the central source is intercepted by cold, bound debris gas within a radius $r_{0}$ and reemitted as thermal radiation with temperatures a few times $10^{4} \mathrm{~K}$. Note that this radius is similar to the radius of the region emitting the $\mathrm{BBB}$. A simple model for the continuum spectrum emitted by a collection of debris clouds orbiting the hole was discussed in $\S 3.5 .2$. The model produces indeed a thermal bump with a flat spectrum at low frequencies $(\alpha>-1)$ turning over at $\sim 10^{15} \mathrm{~Hz}$, and extending to $\sim 3 \mathrm{Ry}$ (emitted by cold matter near $r_{T}$ ). In the previous section it was noted that there is little evidence for absorption in the X-ray spectra of powerful QSOs. This may present a problem for models in which a large fraction of the total energy has been reprocessed by cold gas surrounding the central source.

\subsection{Broad Absorption Lines and Obscured AGN}

Direct evidence for outflow of relatively cold, highly ionized gas from active nuclei with radial velocities up to $0.1 \mathrm{c}$ is provided by the broad, blueshifted absorption lines (BALs) found in the spectra of about $5 \%-10 \%$ of all QSOs (for reviews see Turnshek 1988; Weymann, Turnshek, \& Christiansen 1985). The upper limit to the covering fraction of the outflowing gas, set by the observed amount of resonance line scattering, is comparable to the observed fraction of BALQSOs indicating that the absorbing gas does not cover more than about $10 \%$ of the sky as seen from the central source. All QSOs would then contain a BAL region. It is not clear where the gas is located nor what its relation with other spectral components such as the BLR and the NLR is. A BAL system absorbs at least part of the HIL radiation ( $\mathrm{C} I V, \mathrm{~N} v, \mathrm{Ly} \alpha, \mathrm{Si}$ IV and sometimes also part of the $\mathrm{Mg}$ II line). It must therefore lie outside the BLR (at least outside the HILR) and have a lateral extent larger than the BLR. From the lack of broad [O III] emission from the BAL system it is concluded that the density must be larger than $10^{6-7} \mathrm{~cm}^{-3}$. In combination with the photoionization parameter, which is ill-determined, this constrains the distance from the ionizing source less than $10-100 \mathrm{pc}$. The velocities, densities, and distance to the central source suggest that the BALR is intermediate between the BLR and the NLR. This is supported by the correlation between BAL profile properties and corresponding broad emission line properties.

A standard model BL cloud eclipsing the continuum source would produce sharp absorption features (thermal width $\sim 10$ $\mathrm{km} \mathrm{s}^{-1}$ ) in lines in which it is optically thick. The absence of such lines as well as the lack of X-ray and narrow Ly-edge absorption in QSOs is an important piece of the BLR puzzle and perhaps the best argument in favor of a flattened configuration of the BLR. The broad absorption troughs seen in BALQSOs are clearly not produced by standard broad line clouds. In the tidal disruption model, however, the clouds are much bigger and contain gas with a large range of velocities. Such clouds are expected to produce broad absorption lines. Consider a stellar remnant moving out of the nucleus at $r \sim$ $10 r_{\mathrm{BLR}}$, or $\sim 10^{18.5} \mathrm{~cm}$ for a QSO. Its lateral extent is $0.1 r$, so that it covers the whole BLR for observers in the direction of its velocity vector. Note that the total fraction of the sky covered by remnants around $\sim 10 r_{\text {BLR }}$ is comparable to the covering fraction of the broad emission line region (the number of remnants contributing to the broad line emission is smaller by a factor of 10 , but most of the broad line emission comes from the remnant tails which have larger covering fractions). The density of cold gas in the remnant is $\sim 10^{7-8} \mathrm{~cm}^{-3}$ and the total column density $\sim 10^{22} \mathrm{~cm}^{-2}$, which is smaller than the Strömgren column density (eq. [3.26]). Whenever our line of sight passes through such a highly ionized remnant we will observe broad absorption troughs extending to $\sim v_{0}$, a few 
times $10^{4} \mathrm{~km} \mathrm{~s}^{-1}$ (for black hole masses $10^{8}$ to $10^{9}$ solar masses) blueward of the HILs, with total absorbing column density $\sim 10^{22} \mathrm{~cm}^{-2}$. Complete heating and ionization of the tail of the remnant containing low-velocity gas may explain the occurrence of detached absorption troughs. Multiple absorption troughs may also be observed occasionally.

It is encouraging that stellar remnants just outside the BLR appear able to explain the BAL phenomenon in QSOs. However, if the BAL phenomenon is so intimately related with the broad line region of $A G N$, why are there so few BAL Seyferts? Both the low absorption of X-rays in powerful AGN and the inverse correlation of the $\mathrm{C}$ IV and Ly $\alpha$ equivalent width with continuum luminosity (Baldwin 1977; Kinney, Rivolo, \& Koratkar 1990) suggest that the covering fraction of cold gas in Seyferts is larger than in QSOs. This is also expected in the tidal disruption model if QSOs and Seyferts have similar tidal disruption rates $\left(\sim 1 \mathrm{yr}^{-1}\right)$ while their luminosities are comparable to the Eddington luminosity. Broad absorption lines have been found in only a few percent of all Seyferts observed with IUE (for instance, NGC4151 and NGC3516; see Ulrich 1988). These absorption lines are also blueshifted, but the velocity difference between the emission line peak and the minimum of the absorption line is less than $\sim 2500 \mathrm{~km} \mathrm{~s}^{-1}$, which is considerably smaller than the width of the broad emission lines. There is good evidence that some and perhaps all Seyfert 2 nuclei contain Seyfert 1 nuclei hidden behind obscuring matter, perhaps in the form of an obscuring torus around the nucleus (Antonucci \& Miller 1985). Perhaps not only the covering fraction but also the optical depth of the obscuring matter in Seyferts is larger than in QSOs. This seems plausible in the tidal disruption model since stellar remnants in low-luminosity AGN contain a larger fraction of cold gas than high-luminosity AGN. In such a model BAL QSOs would be the high luminosity counterparts of Seyfert 2 s. In this context it seems interesting that the broad absorption line Seyfert NGC 4151 has been classified as a Seyfert 1.5.

\subsection{The Narrow Line Region}

In the model discussed in $\S 4$ the BLR ends where the stellar remnants become optically thin to the ionizing radiation from the central source. At some distance outside the BLR the outmoving remnants will be slowed down and compressed as they sweep up interstellar matter. In this section we speculate that the narrow line region (NLR), or at any rate its inner part, is formed by such outmoving remnants in their snow plow phase.

The narrow line region extends from $\geq 1 \mathrm{pc}$ to $10-100 \mathrm{pc}$ or more and has been spatially resolved in some nearby Seyferts (see Wilson \& Heckman 1985, and Osterbrock \& Matthews 1986, hereafter O\&M, for recent reviews on the narrow line region). Clavel \& Wamsteker (1989) have recently determined an upper limit for the size of the NLR in the broad line radio galaxy 3C 390.3 of $10 \mathrm{lt}-\mathrm{yr}$. The inner radius of the NLR is therefore not much larger than the outer radius of the BLR. The width of the narrow lines varies from a few $100 \mathrm{~km} \mathrm{~s}^{-1}$ to $1000-2000 \mathrm{~km} \mathrm{~s}^{-1}$. It is correlated with the ionization level of the line and the critical density for collisional deexcitation in the sense that high ionization lines and lines having a higher critical density are broader. The density and velocity dispersion of the narrow line gas appear to increase with decreasing radius in a manner which suggests that the NLR joins smoothly with the BLR. This is supported by the observed correlation between the width of the broad Balmer lines and the narrow [O III] line (Cohen 1983). van Groningen \& de Bruyn (1989, hereafter vG\&dB) have found that the forbidden [O III] line in Seyfert 1 galaxies often has broad wings of $\sim 1000 \mathrm{~km} \mathrm{~s}^{-1}$. They interpret this as due to line emission from a region intermediate between the broad and narrow line regions, a "transition line region" (TLR). This interpretation is supported by a variability study of this line in the Seyfert galaxy Fairall 9 by Stirpe, van Groningen, \& de Bruyn (1989) which indicates that it is emitted in a region at a distance greater than 1 pc from the central source. From observed line ratios vG\&dB conclude that the mean density of the line emitting gas is $\sim 5 \times 10^{6} \mathrm{~cm}^{-3}$. They assume that the TLR material is provided by the outflowing mass from the BLR. The blue asymmetry of the narrow-line profiles indicates radial flow. O\&M give theoretical reasons for preferring decelerated outflow as the dominant motion in the NLR.

The tidal disruption model presents an explanation for the link between BLR and the NLR. In $\S 4.1$ we noted that in Seyferts the outflowing remnants at $\sim 10^{18} \mathrm{~cm}$ are expected to produce some broad [O III] at densities below $\sim 10^{7} \mathrm{~cm}^{-3}$. This may explain the broad [O III] wings found by vG\&dB. What happens at larger radii? Suppose the remnant matter moves through a hot and less dense medium with virial temperature $\sim 10^{7} \mathrm{~K}$. The ram pressure exerted by this medium decelerates the matter and breaks it up by Raleigh-Taylor instability. The terminal velocity of the fragments will be the sound speed in the medium, which is comparable to the width of lines in the NLR. The inner radius of the NLR is then determined by the requirement that a stellar remnant has swept up an amount of mass comparable to its original mass. We may obtain an upper limit to the radius where the NLR begins by assuming that stellar evolution is the dominant source of hot gas in the galactic nucleus, producing $\sim 10^{-4} \lambda$ $(r / \mathrm{pc}) M_{\odot} \mathrm{yr}^{-1}$ within a region of radius $r$, where $\lambda \sim 1$ (see Murphy et al. 1990). We further assume that this gas flows out of the nucleus with velocity $v_{\text {wind }}$. A lower limit to the total amount of interstellar matter swept up by a single remnant at a distance $r$ from the hole may be obtained by multiplying the gas production rate with the dynamical time of the wind and the covering fraction of the remnant yielding

$$
M_{\text {swept }}(r) \geq 2.10^{-3} \lambda\left(\frac{\operatorname{Cov}_{R}}{0.01}\right)\left(\frac{v_{\text {wind }}}{500 \mathrm{~km} \mathrm{~s}^{-1}}\right)^{-1}\left(\frac{r}{\mathrm{pc}}\right)^{2} M_{\odot} .
$$

The swept-up mass exceeds the remnant mass $\frac{1}{2} M_{\odot}$ at a radius $r_{\text {NLR }} \leq 20 \lambda^{-1 / 2} \mathrm{pc}$, which may be identified with the inner radius of the NLR. Note that if the mass produced by stellar evolution would be removed continuously by passing remnants the density of the medium would be lower by a factor $v_{0} / 500 \mathrm{~km} \mathrm{~s}^{-1} \sim 10$, and $r_{\mathrm{NLR}}$ larger by a factor $\sqrt{10}$. We conclude that a narrow line region is expected to be formed at $r \geq(10-100) \lambda^{-1 / 2} \mathrm{pc}$. The density of cold gas at those distances has to be larger than the density given by equation (3.17). The total remnant mass will be $\sim 10^{4} M_{\odot}\left(v_{\text {tid }} / \mathrm{yr}^{-1}\right)$ at the inner radius of the NLR rising to $\sim 10^{6} M_{\odot}\left(v_{\mathrm{tid}} / \mathrm{yr}^{-1}\right)$ at $\sim 100 \mathrm{pc}$. These numbers are in good agreement with the properties of the NLR derived from observations (Wilson \& Heckman 1985).

\subsection{Radio-Loud versus Radio-Quiet $A G N$}

Radio galaxies belong to the class of eliptical galaxies which reside predominantly in regions of high galaxy density, whereas Seyfert nuclei are found in spiral galaxies outside rich clusters of galaxies. Ellipticais are presumably formed via 
merging of galaxies during the formation of groups and clusters of galaxies. This suggests that the population of radioquiet $\mathrm{AGN}$ evolves into radio-loud $\mathrm{AGN}$, and that the difference between the two types is associated with depletion of the gas content of the parent galaxy (or its nucleus) and/or with the growth of the central hole. Cool, partly ionized gas from tidally disrupted stars is an efficient absorber of low-frequency radiation (eq. [3.48]) and may also disrupt and dissipate jets emitted by the central source. Black holes more massive than $M_{\text {crit }} \sim 3 \times 10^{8} M_{\odot}$ do not produce a lot of tidal debris because solar-type stars are swallowed whole. Hills (1978) already suggested that AGN may become radio-loud when the black hole mass exceeds the critical mass. Radio galaxies may derive their energy primarily by electromagnetic extraction of rotational energy from the hole (Blandford \& Znajek 1977), which sends out jets of relativistic particles along its rotation axis. We expect the thermal components in the spectrum, which are due to reprocessing of central nonthermal radiation by cold matter in the nucleus, to be weak or absent. On the borderline of $M \sim M_{\text {crit }}$ we may find radio-loud QSOs and broad line radio galaxies (BLRGs). The permitted lines in BLRGs are indeed very broad and irregular (Miley \& Miller 1979; Antonucci 1984) indicating large black hole masses. The tidal debris model for the BBB and the BLR would be ruled out if these spectral components are also present in the spectra of AGN containing black holes with mass greater than $M_{\text {crit }}$.

\section{CONCLUSIONS}

A direct consequence of the presence of massive black holes in the center of a galactic nucleus is the occasional tidal disruption of a star about once every $10^{3} \mathrm{yr}$ in the vicinity of the hole. The rate of tidal disruptions will be enhanced by perturbations of the gravitational potential in the nucleus, for example during infall of a smaller galaxy. Tidal disruptions may thus provide an important source of fuel for the hole during recurrent periods of enhanced activity induced by galaxy mergers. In hierarchical clustering scenarios for the formation and evolution of galaxies and clusters such merger events are expected to occur most frequently during the early formation and evolution of galaxies and clusters.

Recent studies of the tidal breakup of stars near a massive black hole have shown that the outmoving and freely expanding debris cloud has a wide distribution of binding energies to the hole extending from $-v_{0}^{2}$ to $+v_{0}^{2}$. About half the stellar mass is bound to the hole. It turns around at $r_{0}$ (eq. [1.4]) and will, after circularization on a time scale $\geq T_{0}$ (eq. [1.5]), settle into an accretion torus at $r_{T}$ (eq. 1.1) and be accreted by the hole. The ratio of the largest time scale involved in this process and the time between successive disruptions will determine the amount of fuel stored within $r_{0}$ and also the amplitude of variations in the energy output of the central source on those time scales. Short-term variability may be associated with the dynamical and viscous time scale of the accretion torus. The other half of the debris gas flies out of the nucleus in radially stretched expanding remnants. The radial velocity increases in the radial direction along the remnant from 0 to $\sim v_{0}$ (eq. [1.3]), and the lateral expansion velocity is $v_{*} \sim 500 \mathrm{~km} \mathrm{~s}^{-1}$.

An outmoving and expanding debris cloud heated by the central source in an AGN should break up into two components when the ionization parameter reaches the critical value $\Xi_{c} \sim 10$. Only a small fraction is heated to the Compton temperature. The pressure exerted by the hot component holds up the expansion of the rest of the gas, which breaks up into cold $\left(\sim 10^{4} \mathrm{~K}\right)$ dense clumps or filaments. As the remnant expands further the cold gas gradually passes over into the hot phase while the ionization parameter remains at its critical value. The density of cold gas in outflowing remnants is thus given by the condition $\Xi=\Xi_{c}$ over more than three orders of magnitude in radius. The evolution of bound debris orbiting within $r_{0}$ is more complicated, but it may also break up into a cold cloud surrounded by hot gas as it expands and attempts to spread out over the volume within $\sim r_{0}$. Tidal disruptions of stars thus provides a source of cold, dense gas in near pressure equilibrium with the radiation field from a radius $r_{\mathrm{T}} \sim 10^{-2} r_{0}$ to radii greater than $10^{3} r_{0}$.

There are several important differences between bound and unbound debris gas. Firstly, the spatial distributions are different. The covering fraction of the bound part of a stellar remnant quickly increases after apocenter passage, while the covering fraction of the unbound remnant stays constant. Bound debris tends to spread out within a sphere of radius $\sim r_{0}$ where it may intercept a considerable fraction of the radiation from the central source. The covering fraction of the outmoving, or unbound, part of a stellar remnant increases from 0.01 at the head to $\sim 0.1$ at the tail, depending on the distance and luminosity of the central source. This matter spends only about $100 \mathrm{yr}$ within $1 \mathrm{pc}$ indicating that a large fraction of the radiation from the central source can be intercepted by outmoving remnants within $1 \mathrm{pc}$ only if the tidal disruption rate is $\geq 0.1 \mathrm{yr}^{-1}$. A second important difference concerns the spectrum emitted by bound and unbound debris. Cold dense gas clouds in the vicinity of the central source radiate predominantly as black bodies in thermodynamic equilibrium with the radiation field, whereas lower density gas ( $n_{\text {cold }}$ smaller than $\sim 10^{12} \mathrm{~cm}^{-3}$, eq. [3.40]) at radii greater than $r_{0}$ emits most energy in the lines.

Cold dense clouds near the central source in AGN produce a number of characteristic features in the spectrum (Guilbert \& Rees 1988; Lightman \& White 1988; Ferland \& Rees 1988). The clouds absorb and reflect X-rays, cause Fe K-shell absorption features and reradiate the intercepted energy predominantly as blackbody radiation in the UV and in a $\mathrm{Fe} \mathrm{K}$ fluorescence line. Such absorption and emission features have recently been detected in several Seyferts. These observations indicate that the column densities and ionization parameter of the cold matter are high, as suggested by the tidal debris model (§ 3.4). The tidal debris model further suggests that a large fraction of the energy in the big blue bump is emitted by gas at a distance $\sim r_{0}$ from the central source ( $\left.\$ 3.5 .2\right)$. The low X-ray absorption observed in QSOs may present a problem for models in which the BBB is emitted by a spherically symmetric distribution of clouds with a large covering fraction.

The ratio of line to continuum emission increases with decreasing density. Bound, cold debris gas orbiting at $r \geq r_{0}$ may therefore emit some broad, symmetric recombination lines with characteristic widths $\sim 10^{4} \mathrm{~km} \mathrm{~s}^{-1}$ (Shields 1989). This contribution to the BLR may be most important in lowluminosity AGN since the density of cold debris is fixed by the condition $\Xi=10$. Initially the column density of bound debris from a single star is high, but it will decline quickly as the matter spreads out and that with the highest binding energy is accreted by the hole. This broad line emitting gas should have a distance to the central source of the order of light days (a few times $r_{0} / c$ ). It is tempting to associate this gas with the very broad wings under some high ionization lines in NGC 55448, which are observed to respond to variations in the continuum 
with a time lag of only a few days (Clavel et al. 1990). Intrinsic variations of the gas distribution at $r_{0}$ are expected to occur on a time scale $\sim T_{0}$ and, of course, $v_{\text {tid }}^{-1}$.

The radiative energy (re)emitted by outflowing remnants at radii less than $10^{18} \mathrm{~cm}$ is dominated by recombination lines. The structure of outflowing remnants is discussed in $\S 3.3$ (see Fig. 3). The remnant may consist of three regions: a hot tail, an intermediate region containing both hot and cold gas, and a neutral (cold) region beyond the Strömgren radius. The neutral region is heated mainly by X-rays from the central source and will emit predominantly low ionization lines (Mg II, Fe II, Balmer lines, etc.). The borderline between the different regions shifts outward as the expanding remnant moves away from the central source. The remnant becomes optically thin to the ionizing radiation at $\sim 10^{17.5} \mathrm{~cm}$. This determines the outer boundary of the BLR produced by outmoving remnants. In $\S 4.1$ we have estimated the distribution of the line luminosity with radius for two specific cases (see Fig. 4). The results are the following:

1. The radius and extent of the BLR are consistent with variability studies.

2. The densities, column densities and ionization parameters are in the proper range to explain the observed lines and line ratios in the BLR (Ferland \& Persson 1989).

3. The efficiency of line emission declines rapidly at densities lower than about $10^{8-9} \mathrm{~cm}^{-3}$, which explains the absence of broad [O III] lines.

4. The profile of the broad lines in an individual AGN is the result of the contributions from a limited number of remnants, each having a range of projected radial velocities. The line profiles of individual AGN may therefore have bumps, shoulders and asymmetries, while the average line profile is expected to be approximately logarithmic with a width $\sim v_{0}$.

5 . The covering fraction of outmoving remnants is proportional to the tidal disruption rate and depends also on the continuum luminosity (see eqs. [5.13] and [5.14]). The high tidal disruption rate required to explain the covering fraction in QSOs appears consistent with a high fueling level of the central source. The tidal disruption rate in low-luminosity AGN has to be quite high as well if the broad lines in these sources are emitted by unbound remnant gas. Perhaps bound debris is the dominant source of broad lines in a low luminosity AGN.

Note that there is no confinement problem for the broad line clouds in this model.

The remnant clouds just outside the broad line region have several properties which make them good candidates for the broad absorption systems observed in $\sim 10 \%$ of all QSOs: (1) The chance that our line of sight passes through the head of such a remnant is comparable to the covering fraction of the BLR produced by the line emitting parts of remnants at smaller radii. (2) The lateral extent is large enough to cover the whole BLR. (3) The density and column density are $\sim 10^{7}$ $\mathrm{cm}^{-3}$ and $\leq 10^{22} \mathrm{~cm}^{-2}$, lower than the Strömgren column density, respectively. The remnant will produce deep absorption troughs blueward of the high ionization emission lines. The troughs may extend from $\sim 0 \mathrm{~km} \mathrm{~s}^{-1}$ (or larger if the tail is heated to the Compton temperature) to $v_{0}$. Note that the mass per velocity interval increases with velocity. (4) Narrower absorption lines are expected when our line of sight intersects only part of the remnant.

Models in which the broad emission and absorption line gas is contained in large, randomly distributed and radially outmoving clouds are faced with two problems. First, in some QSOs our line of sight will pass through an optically thick remnant in the BLR $\left(r<10^{18} \mathrm{~cm}\right)$ obscuring the continuum and perhaps part of the BLR. Secondly, why are there no BAL Seyferts? Is the tidal disruption rate in Seyferts too low? In that case the BLR of Seyfert galaxies is probably not due to unbound debris matter. Perhaps BAL Seyferts are not recognized as Seyfert 1s because the absorption is stronger than in BAL QSOs. One might then speculate that Seyfert $2 \mathrm{~s}$ are the low-luminosity counterparts of BAL QSOs.

The kinetic energy and momentum of outflowing remnants are transfered to the interstellar medium at radii $\geq 10 \mathrm{pc}$, which may be identifed with the inner radius of the NLR. The swept up interstellar medium is likely to flow outward with velocity comparable to the sound speed of hot gas in the central region of the galaxy.

Debris clouds from tidally disrupted stars are efficient absorbers of low-frequency radiation. The covering fraction of debris gas decreases with increasing black hole mass. Moreover black holes more massive than $M_{\text {crit }} \sim 10^{8.5} M_{\odot}$ swallow stars whole without producing much debris. Radiative energy produced by such massive holes, perhaps extracted from the rotational energy of a Kerr hole, is not reprocessed by stellar debris. This suggests that the difference between radio-loud and radio-quiet $A G N$ is due to a difference in mass of the central black hole (see also Hills 1978). If AGN containing black holes with masses greater than $M_{\text {crit }}$ have BLRs (and BBBs) similar to AGN having black holes less than $M_{\text {crit }}$ then tidal disruptions are unlikely to be the source of cold gas in AGN.

The main purpose of this paper has been to show that gas from tidally disrupted stars can produce a broad line region similar to that observed in AGN. The results suggest that several other characteristic features in AGN spectra may also be interpreted as due to reprocessing of radiation from the central source by stellar debris. These features include X-ray absorption and line and continuum emission, the big blue bump, broad absorption lines and the narrow line region.

It is a pleasure to thank B. Espey, S. Collin, M. Joly, J. Perry, J. Kaastra, and J. Krolik for discussion, V. Icke and H. Netzer for comments on an earlier version of the manuscript, and $\mathbf{W}$. Shane for careful reading of the manuscript and for giving many useful comments.

\section{REFERENCES}

Antonnucci, R. R. J. A. 1984, ApJ, 281, 112

Antonnucci, R. R. J. A. 1987, in Proc. George Mason University Conference on Supermassive Black Holes, ed. M. Kafatos (Cambridge: Cambridge Univ. Press), 26

Antonnucci, R. R. J. A., \& Miller, J. S. 1984, ApJ, 297, 621

Alloin, D., Pelat, D., Philips, M. M., Fosbury, R. A. E., \& Freeman, K. 1986, ApJ, 308, 23

Arnaud, K. A. et al. 1985, MNRAS, 217, 105

Bahcall, S., \& Tremaine, S. 1988, ApJ, 326, L1
Baldwin, J. A. 1977, ApJ, 214, 679

Bardeen, J. M., \& Petterson, J. A. 1975, ApJ, 195, L65

Blandford, R. D., \& McKee, C. F. 1982, ApJ, 255, 419

Blandford, R. D., \& Znajek, R. L. 1977, MNRAS, 179, 433

Blumenthal, G. R., Faber, S. M., Primack, J. R., \& Rees, M. J. 1984, Nature 311,517

Canizzo, J. K., Lee, H. M., \& Goodman, J. 1990, ApJ, 351, 38

Capriotti, E. R., Foltz, C. B., \& Byard, P. 1980, ApJ, 241, 903

Capriotti, E. R., Foltz, C. B., \& Peterson, B. M. 1982, ApJ, 261, 35 
Carlberg, R. G. 1990, ApJ, 359, L1

Carter, B., \& Luminet, J. P . 1985, MNRAS, 212, 23

Cavalieri, A., \& Padovani, P. 1988, ApJ, 333, L33

Clavel, J., \& Wamsteker, W. 1989, ApJ, 320, L9

Clavel, J., Wamsteker, W., \& Glass, I. 1989, ApJ, 337, 236

(OI Clavel, J., et al. 1991, ApJ, in press

I- Chen, K., Halpern, J. P., \& Filippenko, A. V. 1989, ApJ, 339, 742

Cohen, R. D. 1983, ApJ, 273, 489

Collin-Souffrin, S. 1986, A\&A, 166, 115

Collin-Souffrin, S., Dumont, S., Joly, M., \& Pequignot, D. 1986, A\&A, 166, 27

Collin-Souffrin, S., Dyson, J. E., McDowell, J. C., \& Perry, J. 1988, MNRAS, 232,539

Collin-Souffrin, S., \& Lasota, J. 1988, PASP, 100, 104

Cutri, R. M., Wisniewski, W. Z., Rieke, G. H., \& Lebofsky, M. J. 1985, ApJ, 296,423

Czerny, R. M., \& Elvis, M. 1987, ApJ, 321, 305

Davidson, K., \& Netzer, H. 1979, Rev. Mod. Phys., 51, 715

Edelson, R. A., \& Malkan, M. A. 1986, ApJ, 308, 509

Efstathiou, G., \& Rees, M. J. 1988, MNRAS, 230, 5P

Evans, C. R., \& Kochanek, C. S. 1989, ApJ, 346, L13 (E\&K)

Ferland, G. J., \& Netzer, H. 1984, PASP, 96, 593

Ferland, G. J., \& Persson, S. E. 1989, ApJ, 347, 656

Ferland, G. J., \& Rees, M. J. 1988, ApJ, 332, 141

Frank, J., \& Rees, M. J. 1976, MNRAS, 176, 633

Gaskell, C. M. 1982, ApJ, 263, 79

Gondhalekar, P. M. 1990, MNRAS, 243, 443

Gondhalekar, P. M., O’Brien, P. T., \& Wilson, R. 1986, MNRAS, 222, 71

Guilbert, P. W., \& Rees, M. J. 1988, MNRAS, 233, 475 (G\&R)

Heckman, T. M., Miley, G. K., van Breugel, W. J. M., \& Butcher, M. R. 1981, ApJ, 247, 403

Hernquist, L. 1989, in The Epoch of Galaxy Formation, ed. C.S. Frenk et al. (Dordrecht: Kluwer), 435

Hills, J. G. 1975, Nature, 254, 295

1978, MNRAS, 182517

Joly, M. 1987, in Proceedings of the Rutherford-Appleton Workshop on Emission lines in Active Galactic Nuclei, ed. P. M. Gondhalekar (Oxford: Rutherford Appleton Laboratory, RAL-87-109), 116

Joly, M., Collin-Souffrin, S., Masnau, J. L., \& Nottale, L. 1985, A\&A, 152, 282

Kaastra, J. S., \& Barr, P. 1989, A\&A, 226, 59

Kallman, T., \& Elitzur, M. 1988, ApJ, 328, 523

Kinney, A. L., Rivolo, A. R., \& Koratkar, A. P. 1990, ApJ, 357, 338

Krolik, J., \& Kallman, T. R. 1988, ApJ, 324, 714

Krolik, J., McKee, C., \& Tarter, C. B. 1981, ApJ, 249, 422 (KMT)

Kwan, J., \& Krolik, J. K. 1981, ApJ, 250, 478

Lacey, J. K., Townes, C. H., \& Hollenbach, D. J. 1982, ApJ, 262, 120

Lightman, A. P., \& Shapiro, S. L. 1977, ApJ, 211, 244

Lightman, A. P., \& White, T. R. 1988, ApJ, 335, 57 (L\&W)

Lin, D. N. C., Pringle, J. E., \& Rees, M. J. 1988, ApJ, 328, 103

Lonsdale, C. J., Hacking, P. B., Conrow, T. P., \& Rowan-Robinson, M. 199.0, ApJ, 358, 60

Luminet, J. P., \& Carter, B. 1986, ApJ, 61, 219

Maddox, S. J., Sutherland, W. J., Efstathiou, G., Loveday, J., \& Peterson, B. A. 1990, MNRAS, 247, $1 \mathrm{P}$

Malkan, M. A. 1983, ApJ, 268, 582

Matsuoka, M., Piro, L., Yamauchi, M., \& Murakami, T. 1990, ApJ, 361, 440

Matthews, W. G., \& Capriotti, E. R. 1985, in Astrophysics of Active Galaxies and Quasi-Stellar Objects, ed. J. S. Miller (Mill Valley: University Science Books), 185 (M\&C)

McHardy, I. M. 1989, in Proc. 23rd ESLAB Symp. on Two-Topics in X-ray Astronomy, ed. N. E. White (ESA SP-296), 1111

Miley, G. K., \& Miller, J. S. 1979, ApJ, 228, L55

Miller, J. S., \& Peterson, B. M. 1990, ApJ, 361, 98

Morisawa, K., Matsuoka, M., Takahara, F., \& Piro, L. 1990, A\&A, 236, 299

Murphy, B. W., Cohn, H. N., \& Durisen, R. H. 1990, ApJ, in press

Nandra, K., Pounds, K. A., Stewart, G. C., Fabian, A. C., \& Rees, M. J. 1989, MNRAS, 236, 39P
Netzer, H. 1985, ApJ, 289, 451

1989, in IAU Symp. 134, Active Galactic Nuclei, ed. D. E. Osterbrock \& J. S. Miller (Dordrecht: Kluwer), 69

Noguchi, M. 1988, A\&A, 203, 259

Norman, C. A., \& Scoville, N. 1988, ApJ, 332, 124

O'Brien, P. T., Zheng, W., \& Wilson, R. 1989, MNRAS, 240, 741

Osterbrock, D. E. 1985, in Astrophysics of Active Galaxies and Quasi-Stellar Objects, ed. J. S. Miller (Oxford: Oxford Univ. Press) 1989, Astrophysics of Gaseous Nebulae and Active Galactic Nuclei

(Mill Valley: University Science Books)

Osterbrock, D. E., \& Matthews, W. G. 1986, ARA\&A, 24, 171 (O\&M)

Osterbrock, D. E., \& Shuder, J. M. 1982, ApJS, 49, 149

Padovani, P., Burg, R., \& Edelson, R. A. 1990, ApJ, 353, 438

Peterson, B. M. 1988, PASP, 623, 18

Peterson, B. M., et al. 1990, preprint

Peterson, B. M., \& Ferland, G. J. 1986, Nature, 324, 345 (P\&F)

Petre, R., Mushotzky, R. F., Krolik, J. H., \& Holt, S. S. 1984, ApJ, 280, 499

Pounds, K. A., Stanger, V. J., Turner, T. J., King, A. R., Czerny, B. 1986 MNRAS, 224, 443

Pounds, K. A., Nandra, K., Stewart, G. C., \& Leighly, K. 1989, MNRAS, 240 769

Rafanelli, P. 1985, A\&A, 146, 17

Rees, M. J. 1978, Nature, 275, 516

. 1982, in The Galactic Center, ed. G. Riegler \& R. D. Blandford (New York: AIP), 166 1988, Nature 333,523

Rees, M. J. 1990, Science, 247,817

Rees, M. J., Netzer, H., \& Ferland, G. J. 1989, ApJ, 347, 640

Reichert, G., Petre, R., Mushotzky, R., \& Holt, S. S. 1983, BAAS, 15, 675

Reimers, D., Clavel, J., Groote, D., Engels, D., Hagen, H. J., Naylor, T.

Wamsteker, W., \& Hopp, U. 1989, A\&A, 218, 71

Roos, N. 1981, A\&A, 104, 218 (Paper I) 1985a, ApJ, 294, 479

. 1985b, ApJ, 294, 486

Sanders, D. B., Phinney, E. S., Neugebauer, G., Soifer, B. T. \& Matthews, K. 1989, ApJ, 347, 29

Sanders, R. H. 1984, A\&A, 140, 52

Shields, G. A. 1978, Nature, 272, 706

. 1989, in IAU Symp. 134, Active Galactic Nuclei, ed. D. E. Osterbrock \& J. S. Miller (Dordrecht: Kluwer), 577

Shields, G. A., \& Wheeler, J. C. 1978, ApJ, 222, 667

Stirpe, G. M. 1989, Ph.D. thesis, Leiden Univ. 1990, A\&AS, 85, 1049

Stirpe, G. M., de Bruyn, A. G., \& van Groningen, E. 1988, A\&A, 200, 9

Stirpe, G. M., van Groningen, E., \& de Bruyn, A. G. 1989, A\&A, 211, 310

Tonry, J. L. 1987, ApJ, 322, 632

Turner, T. J., \& Pounds, K. A. 1989, MNRAS, 240, 833

Turnshek, D. A. 1988, in QSO Absorption Lines: Probing the Universe, ed

J. C. Blades, D. A. Turnshek, \& C. A. Norman (Cambridge: Cambridge Univ, Press) 17

Ulrich, M.-H. 1988, MNRAS, 230, 121

van Groningen, E. 1983, A\&A, 126, 363 (vG\&dB)

van Groningen, E., \& de Bruyn, A. G. 1989, A\&A, 211, 293

Wachter, K. W., Strauss, M. A., \& Filippenko, A. V. 1988, ApJ, 330, 91

Wandel, A., \& Yahil, A. 1985, ApJ, 295, L1

Weymann, R. J. A., Turnshek, D. A., \& Christiansen, W. A. 1985, in Astro-

physics of Active Galaxies and Quasi-Stellar Objects, ed. J. S. Miller (Mill

Valley: University Science Books), 333

Wilkes, B. J. 1984, MNRAS, 207, 73

Wills, B., \& Browne, I. W. A. 1986, ApJ, 302, 56

Wilson, A. S., \& Heckman, T. M. 1985, in Astrophysics of Active Galaxies and Quasi-Stellar Objects, ed. J. S. Miller (Oxford: Oxford Univ. Press), 39

Zel'dovich, Ya. B., \& Raizer, Yu. P. 1967, Physics of Shock Waves and High-

Temperature Hydrodynamic Phenomena (London: Academic Press)

Zepf, S. E., \& Koo, D. C. 1989, ApJ, 337, 34 